hep-th/0109069

SLAC-PUB-8984

SU-ITP-00/24

\title{
Linear Sigma Models for Open Strings
}

\author{
Simeon Hellerman, Shamit Kachru, Albion Lawrence and John McGreevy \\ Department of Physics, Stanford University, Stanford, CA 94305 \\ SLAC Theory Group, MS 81, PO Box 4349, Stanford, CA 94309
}

\begin{abstract}
We formulate and study a class of massive $\mathcal{N}=2$ supersymmetric gauge field theories coupled to boundary degrees of freedom on the strip. For some values of the parameters, the infrared limits of these theories can be interpreted as open string sigma models describing D-branes in large-radius Calabi-Yau compactifications. For other values of the parameters, these theories flow to CFTs describing branes in more exotic, non-geometric phases of the Calabi-Yau moduli space such as the Landau-Ginzburg orbifold phase. Some simple properties of the branes (like large radius monodromies and spectra of worldvolume excitations) can be computed in our model. We also provide simple worldsheet models of the transitions which occur at loci of marginal stability, and of Higgs-Coulomb transitions.
\end{abstract}

September 2001 


\section{Introduction}

The study of D-branes wrapped on supersymmetric cycles of Calabi-Yau threefolds serves the dual purpose of providing explicit supersymmetric "brane world" models, and of providing probes of substringy distances in compactifications where quantum geometry comes into its own. To date most calculations of the open string spectra and dynamics in this class of compactifications have been done at particular points in moduli space large radius limits, Gepner points, and CFT orbifold points. Yet a host of important issues require a more global understanding: the behavior of D-branes under topologychanging transitions [1,2], the physics of D-brane probes of closed-string singularities [3], supersymmetry breaking [⿴囗十, vacuum selection, and the stability of BPS states [5, 4, 6, 7, 8]. A description giving even rough features (such as spectra and singularities) of a large class of closed-string backgrounds and D-brane configurations would be of use.

The gauged linear sigma model (GLSM) [9] provides such a description for the type II and heterotic compactifications with only closed strings. Exact CFT descriptions are available only at special points in the moduli space. Instead one constructs a massive $2 \mathrm{~d}$ QFT which has the desired CFT as an infrared fixed point, and with parameters that can be mapped onto coordinates on the CFT moduli space. One may then compute RGinvariant properties (or properties for which the behaviour along the flow is understood) at any point in the moduli space of the Calabi-Yau. Among other things, the GLSM enables one to study simple topology-changing processes in string theory, and to obtain a picture of the "phase" structure that arises as one varies closed string moduli [9, 10, 11]. The appearance of Landau-Ginzburg orbifolds as the small-radius limit of certain Calabi-Yau models [12] is transparent in the GLSM. One may also use these models to find singular CFT points in the moduli space [9:[13].

We can describe D-branes in this picture. Some pieces of this description have been developed independently in [14,15], especially for "A-type" branes wrapping special Lagrangian submanifolds of the CY [16,17], and for some simple "B-type" branes (branes wrapping holomorphic cycles). For B-type branes the physics of the LG phase is undeveloped, so the phase structure of the open string sector is not understood. But the B-type

branes are particularly useful to study since the superpotentials for open-string fields are free from worldsheet instanton corrections [0, 18, 19]. In addition, they are complementary 
to heterotic $(0,2)$ models and F-theory compactifications, in that the data specifying the D-brane configuration consists of sheaves and bundles on a Calabi-Yau background.

In this work we construct linear sigma models for a large class of B-type branes and describe their phase structure.2 The boundary conditions and bundle data are specified by adding degrees of freedom on the worldsheet boundary; they provide the Chan-Paton factors. The boundary couplings provide holomorphic data which specify the D-brane configurations. We will describe a class of D-branes which, when viewed as sheaves on the threefold, arise naturally in $(0,2)$ models [21]. Our discussion is complementary to the heterotic $(0,2)$ GLSMs. For example, the locations of singular CFTs will be different, as we can anticipate from heterotic/type I duality. In the long run, we hope this framework will be useful for studying the variation of the open string spectrum as we move through the open and closed-string moduli space, and for studying in detail the singularity structure of these theories.

The outline of our paper is as follows. In $\S 2$, we write down massive models for branes wrapping B-type cycles with various gauge field backgrounds on the brane, and discuss the phase structure. We postpone technical details until $\S 3$, wherein we describe the relevant supermultiplet structure, boundary conditions, and worldsheet Lagrangians. $\S 4$ presents an alternative technique for branes of finite codimension which is useful for describing Higgs-Coulomb transitions. In $\S 5$, we discuss some applications of our models, including monodromies and marginal stability transitions in closed string moduli space, and branch structures in open string moduli space. In $\S 6$, we develop methods that one can use to compute the spectrum of light fields on the brane worldvolume, and apply this technology to a simple example of a brane on $K 3$. $\S 7$ contains our conclusions. We have put a number of technical details in the appendices. In Appendix A we review the supersymmetry transformations of the bulk multiplets. In Appendix B we review the superspace formalism and introduce superspace for boundary degrees of freedom. In Appendix $\mathrm{C}$ we explain a formula from homological algebra which we will use in $\S 4$.

1 In practice, for space-filling branes wrapping cycles in a compact CY, we will also need to add orientifolds in order to cancel the RR tadpoles. We leave such a description for future work. For a computation of the spectrum of the D-branes at hand, we can imagine that the branes sit at a point in the non-compact spatial directions.

2 Another description of D-branes in different phases, inspired by the GLSM description, can be found in 20]. In $\S 5.1$ we give some results consistent with their analysis. 
The main ideas of this project were presented by S.K. at the Strings 2000 conference in Ann Arbor, Michigan [22]. In the intervening (perhaps overly long) writeup period since then, several papers which have significant overlap with our construction have appeared [23,24,25]. Generalizations of the construction were given in [26].

\section{Physical interpretation and phase structure}

We begin by presenting the crux of the construction, dispensing with technical details until $\S 3$. In this section we assume some familiarity with linear models for closed strings [9].

Our guiding principle in constructing these boundary LSMs is the B-type $\mathcal{N}=2$ supersymmetry which we know we must preserve in the infrared [17]. Since this algebra is nearly identical to $(0,2)$ heterotic supersymmetry, the multiplet and interaction structure we employ will be for the most part familiar from studies of heterotic LSMs [21,27].

Our approach to boundary conditions differs from previous work on this subject. The virtue of a linear sigma model is its trivial UV field space. All of the nonlinearities are encoded in the action, and the nonlinear sigma model arises upon RG flow. We adopt this philosophy in describing boundary conditions; we add boundary potentials for bulk fields, and interactions between bulk and boundary fields. We can do this in a manifestly B-type supersymmetry invariant way, before imposing boundary conditions. The resulting boundary equations of motion of the bulk fields should be satisfied as boundary conditions. In the absence of boundary interactions, a scalar will satisfy the Neumann condition that the boundary value is free. To make a scalar with a Dirichlet condition, $S(\phi)=0$, say, we find a supersymmetric way to add to the action a potential on the boundary $\partial \Sigma$ of the worldsheet:

$$
\int_{\partial \Sigma}|S|^{2}
$$

As in [28], this potential term dominates the boundary equation of motion in the infrared.

We will first examine extreme limits of the GLSM parameters, where a good approximation to the infrared physics arises from studying the vacua and light fluctuations evident in the classical worldsheet action. These limits include the large-radius CY, and the "very small radius" Gepner point, as identified in [9] and reviewed below. In these limits we study the vacuum manifold and the spectrum of massless boundary fermions. The vacuum manifold will specify the background CY geometry and the submanifold on which 
the branes are wrapped; the spectrum of massless fermions will identify the Chan-Paton bundle on these branes.

To be concrete, we will study D-branes on the quintic CY in $\mathbb{P}^{4}$ for which the bulk $(2,2)$ linear sigma model is well-known [9]. The field content is: one $U(1)$ gauge multiplet, five chiral multiplets $\phi^{i}$ with charge 1 , and one chiral multiplet $p$ of charge -5 . These fields are coupled via a quasihomogeneous bulk superpotential, $W=p G(\phi)$, of degree 5 in the $\phi$ 's.

\subsection{Boundary fields}

We want to model a D-brane wrapped on a supersymmetric cycle of the quintic, with some gauge bundle $V$. Let the cycle be $\mathcal{S}=\left\{S^{A}(\phi)=0, \forall A=1 \ldots l\right\}$, a transverse complete intersection. To do this, add the following matter fields at the boundary of the worldsheet. 3 We use $l$ boundary Fermi multiplets $\gamma_{A}$, with charges $d^{A}=-\operatorname{degree}\left(S^{A}(\phi)\right)$ (which will allow us to cut out the codimension $l$ cycle of the CY), and $r+1$ boundary Fermi multiplets $\beta_{a=1 \ldots r+1}$, with charges $n_{a}$ (states of which will supply the Chan-Paton factors). As in $(0,2)$ models, a fermi multiplet consists of a complex fermion and a complex auxiliary boson. We denote the auxiliary partners of $\gamma_{A}, \beta_{a}$ by $g_{A}, b_{a}$, respectively. The Fermi superfields satisfy the chiral constraints:

$$
\begin{aligned}
& Q^{\dagger} \gamma_{A}=0 \\
& Q^{\dagger} \beta_{a}=0
\end{aligned}
$$

where $Q^{\dagger}$ is one of the B-type supercharges preserved by the boundary theory (see Appendix A for a definition of the supersymmetry transformations). We also use a boundary chiral multiplet $\wp$ with charge $-m$. This multiplet is not familiar from $(0,2)$ supersymmetry; it consists of a bosonic component $\wp$ and an auxiliary fermion component $\xi$ (see $\S 3)$. The short boundary multiplets only differ in the statistics of their lowest component. They contain the same number of degrees of freedom and obey

$$
\left\{\beta, \beta^{\dagger}\right\}=1, \quad\left[\wp, \wp^{\dagger}\right]=1
$$

The important interaction term for these fields is the boundary superpotential:

$$
\int_{\partial \Sigma} \int d \theta\left(\gamma_{A} S^{A}(\phi)+\wp F^{a}(\phi) \beta_{a}\right)
$$

3 We label supermultiplets by their lowest component. 
(boundary superspace is defined in Appendix B). $S^{A}$ is a homogenous polynomial in $\phi$ of degree $d^{A}$; while $f^{a}$ is a homogenous $\left(m-n_{a}\right)$ th degree polynomial in $\phi$. In components, this amounts to

$$
\int_{\partial \Sigma}\left(g_{A} S^{A}(\phi)+b_{a} \wp F^{a}(\phi)+\gamma_{A} \partial_{i} S^{A}(\phi) \Theta^{i}+\beta_{a}\left(\wp \partial_{i} F^{a}(\phi)+\xi F^{a}(\phi)\right)\right) .
$$

where the $\Theta$ s are the superpartners of the bulk $\phi$ fields.

The $U(1)$ symmetry acting only on boundary fields

$$
\begin{gathered}
\beta_{a} \mapsto e^{i \alpha} \beta_{a} \\
\wp \mapsto e^{-i \alpha} \wp
\end{gathered}
$$

preserves the interaction (2.3). In spacetime, it acts as the center-of-mass $U(1)$ symmetry of the D-brane configuration. We will gauge this symmetry, and project onto the sector of states of the boundary theory with unit charge.

\subsection{Review of bulk phase structure}

The IR fixed point governing the bulk quantum field theory will be the same as in the $\mathcal{N}=(2,2)$ case. For the reader's convenience we review the story [9] for the quintic: this captures many of the essential features for CY hypersurfaces and complete intersections in more general toric varieties. With $\phi_{i}, p$ the scalars in the chiral multiplets, and $\sigma$ the complex scalar in the vector multiplet, the bosonic potential in the bulk has the form:

$$
U_{k i n}=|G(\phi)|^{2}+|p|^{2} \sum_{i}\left|\frac{\partial G}{\partial \phi_{i}}\right|^{2}+\left(\sum_{i}\left|\phi_{i}\right|^{2}-5|p|^{2}-r\right)^{2}+|\sigma|^{2}\left(\sum_{i}\left|\phi_{i}\right|^{2}+25|p|^{2}\right)
$$

where the first two terms arise from the superpotential; the third term arises from the $D^{2}$ term after integrating out the auxiliary field $D$; and the final term is related by supersymmetry to the gauge-covariant kinetic term.

In semiclassical regimes, the CFT is determined by the vacuum manifold and the fluctuations around it. At large positive $r$, the $D^{2}$ term requires that some of the $\phi$ s are large and nonzero. The first term in (2.6) requires $G=0$. Since some $\phi^{i}$ are nonvanishing, not all $\partial_{i} G$ can vanish for $G$ transverse, so $p=\sigma=0$ and their fluctuations are massive. Since $p=0$, the D-term equation plus the $U(1)$ gauge symmetry forces the $\phi$ s to live in $\mathbb{P}^{4}$. The equation $G=0$ forces $\phi$ to live on the quintic hypersurface in $\mathbb{P}^{4}$. As $r \rightarrow \infty, r$ can be identified with the Kähler class of the quintic CY. Since $r, \phi$, and the gauge coupling $e^{2}$ are large in the IR, the fields transverse to the vacuum manifold are very massive. 
At large negative $r, p$ must be non-zero due to the $D^{2}$ term. Thus $\sigma=G=\partial_{i} G=0$.

Since $G$ is transverse, $\phi^{i}=0$ and $|p|=\sqrt{-r / 5}$. The $\phi^{i}$ are not massive but have a superpotential $G$. The vev of $p$ breaks the $U(1)$ gauge symmetry to a $\mathbb{Z}_{5}$ which rotates $\phi$ by fifth roots of unity. Thus the theory in this phase is a Landau-Ginzburg orbifold. The limit $r \rightarrow-\infty$, for $G=\sum_{i}\left(\phi^{i}\right)^{5}$, is conjectured to be an exactly solvable CFT [29].

\subsection{The Calabi-Yau phase}

To determine which D-brane configuration we are making, and identify the GLSM parameters with moduli of the infrared CFT, we consider the large-radius CY phase of the LSM. After integrating out auxiliary bosons, the potential energy at the boundary of the string is

$$
\sum_{A}\left|S^{A}(\phi)\right|^{2}+\sum_{a}\left|\wp F^{a}(\phi)\right|^{2}
$$

The infrared theory will describe fluctuations about the supersymmetric vacuum, in which this potential will vanish. We start by setting $S^{A}(\phi)=0$. The fact that the CY coordinates satisfy this constraint at the boundaries of the string in the IR indicates that we are describing a D-brane wrapped on the algebraic cycle $\mathcal{S}$.

Next, suppose that the $F^{a}$ are chosen so that they do not have a simultaneous zero on the quintic. This forces $\wp=0$ (and leaves $\wp$ with only massive fluctuations). $F^{a}$ also gives a mass to a particular linear combination of the boundary fermions $\beta^{a}$ through the nonvanishing mass term:

$$
-\sqrt{2} \beta_{a} F^{a} \xi
$$

Meanwhile, $\gamma_{A}$ pair with the bulk fermions normal to $S=0$ via the interaction

$$
-\sqrt{2} \gamma_{A} \partial_{i} S^{A} \Theta^{i}
$$

The massless fermions will transform in some vector bundle over $\mathcal{S}$. This bundle arises exactly as in heterotic $(0,2)$ models [9,21]. The functions $F^{a}$ are homogenous polynomials of order $m-n_{a}$. If we choose a section $s_{a}$ of $\oplus \mathcal{O}\left(n_{a}\right)$ over $\mathbb{P}^{4}$, then $F^{a}$ will provide a map to sections of $\mathcal{O}(m)$ by contraction of indices. The bundle $\tilde{V}$ over $\mathbb{P}^{4}$ is then defined by the following exact sequence:

$$
0 \rightarrow \tilde{V} \rightarrow \bigoplus_{a=1}^{r+1} \mathcal{O}\left(n_{a}\right) \rightarrow \mathcal{O}(m) \rightarrow 0
$$


$\tilde{V}$ is the kernel of the map given by $F^{a} ; V$ is the restriction of this bundle to $\mathcal{S}$. The charge- $n_{a}$ fermions $\beta_{a}$ are sections of $\mathcal{O}\left(n_{a}\right)$, restricted to $\mathcal{S}$. The massless fermions are in the kernel of $F^{a}$ and therefore live in the bundle $V$.

If the massless boundary fermions transform as sections of $V$, the Hilbert space of states that they create will transform in a $2^{r}$-dimensional reducible representation of the structure group. In flat space with a trivial bundle, in order to select out Chan-Paton states in the fundamental representation, we would project onto states with precisely one fermion excitation. The analogue in our Calabi-Yau model is to project onto states which carry the correct charge $(+1)$ under the boundary symmetry (2.5). This projection and its implementation will be further discussed in $\S 3$.

At least some of the open string moduli are manifest in this description of the D-brane. Changing the $S^{A}(\phi)$ moves the cycle $\mathcal{S}$ in its moduli space, while perturbing the $F^{a}(\phi)$ corresponds to moving in the moduli space of bundles $V \rightarrow \mathcal{S}$.

\subsection{The Landau-Ginzburg phase}

When $r$ is large and negative, the bulk theory is a Landau-Ginzburg orbifold. The fluctuations of $\phi$ will be governed by the bulk and boundary potentials. All of the boundary fermions are massless in this phase.

When $F$ is set to zero there is no mass term for $\wp$ in the action. However, the boundary symmetry projection allows only a finite number of states of the $\wp$ field; the target space does not develop a noncompact branch. This is explained in greater detail in $\S 5.2$, and we work out the spectrum of states in an example in $\S 6$.

\subsection{A few words about quantum corrections}

The LSM is most useful in regimes where a semiclassical expansion is valid. One can then reliably identify the light excitations in the $2 \mathrm{~d}$ field theory, and the corrections obtained by integrating out bulk massive modes are suppressed by powers of $1 /|r|$. The massive boundary modes constitute a finite number of degrees of freedom and therefore their effects are computable. An argument along the lines of [30] indicates that the boundary superpotential is not renormalized.

Because we have identified good candidates for the supercharges and global $U(1)$ symmetries in the infrared theory, as in [9, 31, 13, 32] certain quantities can be evaluated reliably in the massive theory. For instance, changing $r$ is a $Q$-exact operation in the Bmodel [33]. Therefore, chiral operators in the $Q$-cohomology of the B-model should have 
$r$-independent properties, which can be studied without loss of generality in the regimes where the semiclassical expansion is good. The simplest example for closed strings is the part of the chiral ring [34] which is visible in the B-model. The open string analogue of this is the spectrum of massless fermionic open string states stretched between D-branes (or the corresponding spectrum of boundary-condition changing operators), which is computable in topological open string theory.

Similarly, we can calculate the spectrum of massless fermionic open string states and the spacetime superpotential which governs them. This coupling is $r$ independent and has been computed (through its correspondence with deformation theory of curves) in various simple geometric situations in [5, 18, 19]; these computations are discussed in the framework of open string field theory in [35]. It should be possible to set up the calculation of these amplitudes directly in the LSM; analogous closed-string calculations in the LSM framework are discussed in e.g. [36, 13].

There is some $r$-dependent information of interest: for example, the phase structure, monodromy matrices, local behavior at marginal stability transitions, and features of brane-antibrane systems (c.f. $\S 5$ and [37]). Even in the absence of an analog of the closed string half-twisted model, it seems likely that in semiclassical regimes other properties of the CFT should be calculable using the linear model.

\section{Ingredients and Details}

We now describe our construction in detail. For concreteness we phrase our discussion in the context of the quintic CY.

In order to describe strings ending on D-brane configurations preserving $4 \mathrm{~d} \mathcal{N}=1$ supersymmetry, the IR fixed point of our massive theory must have $\mathcal{N}=2$ superconformal symmetry. Furthermore, since closed strings propagating away from the D-branes will see a background preserving $4 \mathrm{~d} \mathcal{N}=2$ supersymmetry, the bulk action of the worldsheet should have $\mathcal{N}=(2,2)$ superconformal symmetry broken to $\mathcal{N}=2$ superconformal symmetry by the boundary theory.

We begin with a massive $\mathcal{N}=2$ theory with four supersymmetries, half of which are preserved by the worldsheet boundary. We assume that these flow to the desired IR supersymmetries. The bulk multiplets have been described in [9], and consist of a vector multiplet $\left(v_{\alpha}, \lambda_{ \pm}, \sigma, D\right), 5$ chiral multiplets of gauge charge $1,\left(\phi^{i}, \psi_{ \pm}^{i}, F^{i}\right)$ with $i=1 \ldots 5$, and a chiral multiplet of charge $-5,\left(p, \psi_{ \pm}^{p}, F^{p}\right)$. Here $\phi$ is a complex scalar; $\psi$ 
a complex fermion; $F$ a complex auxiliary scalar; $v_{\alpha}$ a 2 d vector field with field stregth $v_{+-}=\frac{i}{q}\left[P_{+}, P_{-}\right] ; \lambda$ a complex fermion; $\sigma$ a complex scalar; and $D$ a real auxiliary field. Their transformations under the bulk $\mathcal{N}=2$ supersymmetry algebra are reviewed in Appendix A.

We work on the infinite strip parametrized by the time coordinate $x_{0}$ and the spatial coordinate $x_{1} \in[0, \pi]$. We study B-type boundary conditions, which respect the half of the $(2,2)$ supersymmetry transformations generated by $Q$ and $Q^{\dagger}[17$.

\subsection{B-type supersymmetric bulk terms}

By adding boundary terms it is possible to write full-superspace bulk terms in a way which is manifestly invariant under the B-type supersymmetry which we wish to preserve. For a gauge-invariant bulk operator $\mathcal{O}$,

$$
\int d^{4} \theta \mathcal{O}=\frac{1}{8} Q Q^{\dagger}\left[S, S^{\dagger}\right] \mathcal{O}+\partial_{0} X+\partial_{1} Y .
$$

Here $S$ and $S^{\dagger}$ are the supercharges which are broken by the boundary theory, and $X$ and $Y$ are gauge invariant operators. The time derivative term is irrelevant for our purposes, while $Y$ gives a contribution to the boundary action. Thus, by rearranging the order in which we act with the bulk supercharges, we can render a full-superspace integral manifestly B-type supersymmetry invariant.

For a chiral multiplet $\phi$ of charge $q$, the B-type supersymmetric action is as follows (using the transformations given in Appendix A). Let us define linear combinations of $\psi_{ \pm}$ using the notation used for the B-twisted topological sigma model [33]:

$$
\begin{aligned}
\eta & =\frac{1}{\sqrt{2}}\left(\psi_{+}+\psi_{-}\right) \\
\Theta & =\frac{1}{\sqrt{2}}\left(\psi_{+}-\psi_{-}\right) .
\end{aligned}
$$

The kinetic terms for the $\phi$ multiplets can be written as

$$
\begin{aligned}
\frac{1}{8} Q Q^{\dagger}\left[S, S^{\dagger}\right] \phi \phi^{\dagger}= & \partial_{0}(\ldots)+ \\
& \frac{i}{2}\left(\tilde{\nabla}_{1} \Theta \eta^{\dagger}-\eta\left(\tilde{\nabla}_{1} \Theta\right)^{\dagger}+\eta \nabla_{0} \eta^{\dagger}+\Theta \tilde{\nabla}_{0} \Theta^{\dagger}\right) \\
& +\frac{1}{4} F F^{\dagger}+\tilde{\nabla}_{0} \phi \nabla_{0} \phi^{\dagger}-\tilde{\nabla}_{1} \phi\left(\nabla_{1} \phi\right)^{\dagger}-\frac{q}{\sqrt{2}} D|\phi|^{2} \\
& +q\left(\frac{1}{2} \phi\left(\left(\lambda_{-}^{\dagger}+\lambda_{+}^{\dagger}\right) \eta^{\dagger}+\left(\lambda_{-}^{\dagger}-\lambda_{+}^{\dagger}\right) \Theta^{\dagger}\right)+\text { h.c. }\right) \\
& \frac{i q}{2} \phi \phi^{\dagger}\left(\partial_{+} \sigma^{\dagger}+\partial_{-} \sigma\right) .
\end{aligned}
$$


Here

$$
\begin{aligned}
\nabla_{0} \phi & \equiv \frac{i}{2}\left\{Q, Q^{\dagger}\right\} \phi & \nabla_{1} \phi & \equiv \frac{i}{2}\left\{Q, S^{\dagger}\right\} \phi \\
\tilde{\nabla}_{0} \phi & \equiv \frac{i}{2}\left\{S, S^{\dagger}\right\} \phi & \tilde{\nabla}_{1} \phi & \equiv \frac{i}{2}\left\{Q^{\dagger}, S\right\} \phi .
\end{aligned}
$$

Note that $\nabla_{1}$ is not anti-hermitean.

For the vector multiplet kinetic terms we get

$$
\begin{aligned}
\frac{1}{8} Q Q^{\dagger}\left[S, S^{\dagger}\right] \sigma \sigma^{\dagger} & =\partial_{0}(\ldots)-\partial_{+} \sigma^{\dagger} \partial_{-} \sigma-\frac{1}{2} D^{2}-\frac{1}{4} v_{+-}^{2} \\
& -i\left(\lambda_{+} \partial_{-} \lambda_{+}^{\dagger}+\lambda_{-} \partial_{+} \lambda_{-}^{\dagger}\right) \\
& +\frac{1}{2} \partial_{1}\left\{\sigma\left(-\partial_{+} \sigma^{\dagger}-\frac{1}{\sqrt{2}}\left(i D-\frac{v_{+-}}{\sqrt{2}}\right)\right)+\sigma^{\dagger}\left(\partial_{-} \sigma+\frac{1}{\sqrt{2}}\left(i D+\frac{v_{+-}}{\sqrt{2}}\right)\right)\right. \\
& \left.+i\left(\lambda_{+}^{\dagger} \lambda_{+}-\lambda_{-}^{\dagger} \lambda_{-}\right)\right\} .
\end{aligned}
$$

The bulk Fayet-Iliopoulos (FI) term and the worldsheet theta term can also be made manifestly invariant under B-type supersymmetry. Take the term:

$$
\sqrt{2} Q Q^{\dagger} \sigma=D+\frac{i}{2} v_{+-}-\sqrt{2} i \partial_{1} \sigma .
$$

Let

$$
t=i r+\frac{\theta}{2 \pi}
$$

Then

$$
\text { (it) } \sqrt{2} Q Q^{\dagger} \sigma+h . c .=-r D-\frac{\sqrt{2} \theta}{4 \pi} v_{+-}+\frac{\sqrt{2} \theta}{2 \pi} \partial_{1}\left(\sigma+\sigma^{\dagger}\right)+\sqrt{2} r i \partial_{1}\left(\sigma-\sigma^{\dagger}\right) .
$$

The bulk superpotential term, however, cannot be written in a manifestly B-type supersymmetry invariant way (as far as we can tell). Following the above strategy, it can be written as:

$$
-i Q S W(\phi)+\text { h.c. }=-\partial_{i} W F^{i}+\partial_{i} \partial_{j} W \eta^{i} \Theta^{j}+h . c
$$

Acting on this with $Q^{\dagger}$ gives

$$
-i Q^{\dagger} Q S W \propto \partial_{1}(Q W)
$$

which gives a nonvanishing boundary term. This is known as the "Warner problem," since it was pointed out in [38]; we discuss it further in $\S 3.7$ 目.

4 This issue was studied recently in [39]. 


\subsection{Boundary multiplets}

In this section we will describe multiplets of B-type supersymmetry which live on the boundary of the string.

\section{Boundary vector multiplets}

There are two candidates for a boundary vector multiplet. There is a real multiplet, with superspace expansion

$$
s+\sqrt{2} \theta \lambda-\sqrt{2} \bar{\theta} \lambda^{\dagger}+\theta \bar{\theta} d
$$

where $s$ is a real scalar, $d$ is a real auxiliary boson, and $\lambda$ is a complex fermion. For B-type supersymmetry, this multiplet only arises in the reduction of the bulk vector multiplet.

We may also define a real supersymmetry singlet. We shall refer to this as a boundary vector multiplet. It is just a boundary gauge field $a_{0}$ which is annihilated by all supercharges; we will include such a multiplet to gauge the boundary symmetry (2.5).

\section{Fermi multiplets}

We can define boundary Fermi multiplets following the discussion of $(0,2)$ models in [9]. They consist of a boundary fermion $\gamma$ and a boundary auxiliary field $g$. Suppose $\gamma$ has charge $q$ under a boundary vector multiplet $v$. The superfield satisfies the chiral constraint

$$
Q^{\dagger} \gamma=E
$$

where $E$ is any boundary chiral boson $\left(Q^{\dagger} E=0\right)$ with charge $q$ and components $\left(E, \psi_{E}\right)$. For example, $E$ can be a function of the boundary values of the bulk chiral fields.

The supersymmetry transformations of this multiplet are:

$$
\begin{aligned}
& Q \gamma=g \quad Q^{\dagger} \gamma=E \\
& Q g=0 \quad Q^{\dagger} g=-2 i \nabla_{0} \gamma+i \xi_{E} .
\end{aligned}
$$

\section{Boundary chiral multiplets}

Boundary chiral multiplets consist of a complex scalar $\wp$ and a complex fermion $\xi$. There is no auxiliary boson. The superfield satisfies the chiral constraint

$$
Q^{\dagger} \wp=\tau
$$

where $\left(\tau, g_{\tau}\right)$ is a fermi multiplet of the same charge as $\wp$ satisfying $Q^{\dagger} \tau=0$. The supersymmetry transformations are:

$$
\begin{aligned}
Q \wp=-i \xi & Q^{\dagger} \wp=-i \tau \\
Q \xi=0 & Q^{\dagger} \xi=2 \nabla_{0} \wp-g_{\tau}
\end{aligned}
$$

We will see that the fermion $\xi$ will in general have no kinetic terms and will be massive; it can be integrated out algebraically. 


\subsection{Boundary terms in the action}

In this section we describe supersymmetric actions for boundary fields, including their couplings to the bulk chiral and vector multiplets.

\section{Kinetic terms}

As in [26] we use first-order kinetic terms for short boundary multiplets of both statistics. Assume $E$ is a holomorphic function of boundary chiral superfields $\chi^{I}$. Then the following kinetic term is supersymmetric:

$$
\begin{aligned}
S_{\text {fermi }}= & \int d x^{0} d^{2} \theta \gamma^{\dagger} \gamma \\
= & \int d x^{0}\left[i\left(\gamma^{\dagger} \nabla_{0} \gamma-\nabla_{0} \gamma^{\dagger} \gamma\right)\right. \\
& \left.-i \gamma^{\dagger} \partial_{I} E(\chi) \xi_{\chi}^{I}-i \gamma \bar{\partial}_{I} E^{\dagger}\left(\chi^{\dagger}\right) \xi_{\chi}^{I \dagger}+|g|^{2}-|E|^{2}\right]
\end{aligned}
$$

For a boundary chiral multiplet $\wp$, we add a magnetic field term

$$
\mathcal{L}=\int d^{2} \theta B \wp^{\dagger} \wp=B\left(i\left(\nabla_{0} \wp\right) \wp^{\dagger}-i \wp\left(\nabla_{0} \wp^{\dagger}\right)-\xi \xi^{\dagger}+\tau \tau^{\dagger}-i\left(g_{\tau} \wp^{\dagger}-g_{\tau}^{\dagger} \wp\right)\right)
$$

A second order kinetic term for a boundary chiral multiplet is less relevant and therefore flows away in the IR; we omit it from the outset. Canonical quantization then gives

$$
\left[\wp, \wp^{\dagger}\right]=1 / B
$$

The coupling to the magnetic field masses up the fermion $\xi$ and halves the number of $\wp$ degrees of freedom. The set of states made by $\wp$ is the Hilbert space of a harmonic oscillator. The number operator $\wp^{\dagger} \wp$ for this oscillator is now the generator of phase rotations of $\wp$. The $\wp$ multiplet becomes the bosonic analog of a Fermi multiplet. Henceforth, we normalize $\wp$ so that $B \equiv 1$.

\section{Superpotential terms}

The crucial supersymmetry invariant for our purposes is the boundary superpotential term, which is an integral over half of the B-type superspace. We will use terms of this form to specify our brane configuration and its gauge bundle. Take a set of Fermi multiplets $\gamma_{A}$,

such that $Q^{\dagger} \gamma_{A}=E_{A}(\chi)$, with $\chi$ representing any scalar multiplet on the boundary with 
$Q^{\dagger} \chi^{I}=\tau^{I}$. Given a collection of holomorphic functions $S^{A}(\chi)$ of the scalar multiplets, the object

$$
\begin{aligned}
S_{\text {super }} & =\int d x^{0} d \theta \sum_{A} \gamma_{A} S^{A}(\chi)+\text { h.c. } \\
& =\sum_{A} \int d x^{0}\left[\sum_{I} g_{A} S^{A}+i \partial_{I} S^{A} \gamma_{A} \xi_{\chi}^{I}\right]+\text { h.c. }
\end{aligned}
$$

is invariant under supersymmetry if

$$
Q\left(E_{A} S^{A}-\gamma_{A} \partial_{I} S^{A} \tau^{I}\right)=0
$$

This is easily satisfied if $E_{A} S^{A}$ is a constant and if $S^{A}$ is chiral; this will be the case for all of the examples we consider. Upon integrating out the auxiliary bosons, $g_{A}$, one finds a boundary potential

$$
V=\left|S^{A}(\wp)\right|^{2}
$$

\subsection{Boundary conditions}

Next, we work out the implications of the boundary terms in $\S 3.1$ for the boundary values of the bulk fields.

Consider first the case with no boundary superpotential. Then, the variation of the bulk action under variation of $\phi^{\dagger}$ at the boundary is

$$
\int_{\partial \Sigma}\left(-D_{1} \phi\right) \delta \phi^{\dagger} .
$$

Thus, since we allow arbitrary variations of $\phi^{\dagger}$ at the boundary, we find

$$
\left.D_{1} \phi\right|_{\partial \Sigma}=0
$$

If the bulk action is B-type supersymmetric (c.f. $\S 3.1$ ), the boundary conditions on fermions will be the ones implied by supersymmetry from the conditions on the bosons. The boundary equation of motion for $\Theta^{\dagger}$ from $(3.3)$ is

$$
0=\int_{\partial \Sigma} \delta \Theta^{\dagger} \eta
$$

Since $\delta \Theta^{\dagger}$ is arbitrary,

$$
\left.\eta\right|_{\partial \Sigma}=0
$$


and in particular there is no $\eta$ zero mode, as we would expect from e.g. Neumann conditions on a superstring in flat space [40]. This is consistent with the fact that

$$
\left.\left.Q \eta\right|_{\partial \Sigma} \sim D_{1} \phi\right|_{\partial \Sigma}
$$

Next we explain how boundary superpotentials effect supersymmetric Dirichlet conditions. Add a boundary superpotential of the form

$$
\int_{\partial \Sigma} d \theta \gamma S(\phi)
$$

Upon addition of such a boundary superpotential, the boundary equation of motion for a bulk scalar $\phi^{i}$ becomes

$$
\begin{array}{r}
\int d x^{0} \delta \phi^{i}\left(\eta_{i \bar{\jmath}} D_{1} \phi^{\bar{\jmath}}+g_{A} \partial_{i} S^{A}-\partial_{i} E_{A} E_{A}^{\dagger}+i \gamma_{A}^{\dagger} \partial_{i} \partial_{I} E_{A} \xi^{I}-i \partial_{i} \partial_{I} S^{A} \gamma_{A} \xi^{I}\right) \\
=\int d x^{0} \delta \phi^{i}\left(\eta_{i \bar{\jmath}} D_{1} \phi^{\bar{\jmath}}-\partial_{i} S^{A} S^{A \dagger}-\partial_{i} E_{A} E_{A}^{\dagger}+\text { fermions }\right)
\end{array}
$$

(In the second step we have integrated out the boundary auxiliary bosons.) In the UV these are some "rotated" boundary conditions. In the IR the dominant terms come from the bulk and boundary potentials and the derivative terms in (3.27) can be ignored. This is as in 28]. Fluctuations in directions in field space $n^{i}$ with

$$
n^{i} \frac{\partial S}{\partial \phi_{i}} \neq 0 \quad \text { or } \quad n^{i} \frac{\partial E}{\partial \phi_{i}} \neq 0
$$

will be energetically forbidden; they are effectively frozen to zero by Dirichlet boundary conditions in the IR. On the other hand, in field space directions for which $\frac{\partial}{\partial \phi_{i}} S$ and $\frac{\partial}{\partial \phi_{i}} E$ both vanish, the boundary equation of motion will impose the Neumann condition $\left.D_{1} \phi^{i}\right|_{\partial \Sigma}=0$ as usual. In this manner, the IR dynamics yield effective Dirichlet conditions, $\left.S(\phi)\right|_{\partial \Sigma}=\left.E(\phi)\right|_{\partial \Sigma}=0$, with Neumann conditions in all other directions.

When we add (3.26) the variation of the action with respect to $\Theta^{\dagger}$ is:

$$
0=\int_{\partial \Sigma} \delta \Theta_{i}^{\dagger}\left(\eta_{i}-\gamma \frac{\partial}{\partial \phi^{i}} S(\phi)\right)
$$

Thus, the boundary fermion $\gamma$ supplies the zero mode for $\eta$ normal to the hypersurface $\{S(\phi)=0\}$. This zero mode is required by supersymmetry if the bosonic partner has Dirichlet conditions. 
Boundary equations of motion for the bulk vector multiplet are as follows. The variation of $v_{0}$ gives

$$
0=\delta S=\int_{\partial \Sigma} \delta v_{0}\left(-v_{+-}-\theta+\left.j_{0}\right|_{\partial \Sigma}\right),
$$

where $j_{0}$ is the gauge current coupling to $v_{\alpha}$.

The boundary variation of $\sigma, \sigma^{\dagger}$ gives

$$
\int_{\partial \Sigma}\left(\frac{1}{2} \partial_{1} \sigma+\frac{1}{\sqrt{2}}\left(2 i r+2 \theta+\frac{i}{2} D+\frac{v_{+-}}{2 \sqrt{2}}\right)\right) \delta \sigma^{\dagger}-\frac{1}{2} \sigma \partial_{1} \delta \sigma^{\dagger}+\text { h.c.. }
$$

We note here that the vanishing of this term is consistent with the A-type boundary condition

$$
\sigma-\left.e^{i \gamma} \sigma^{\dagger}\right|_{\partial \Sigma}=0
$$

with Neumann conditions on the orthogonal combination. The angle $\gamma$ that this line in the complex $\sigma$ plane makes with the real axis is unfixed.

Finally, the equations of motion together with the supersymmetry variations of $\sigma$ require that $\lambda$ satisfy the boundary condition

$$
e^{i \gamma} \lambda_{+}+\left.\lambda_{-}\right|_{\partial \Sigma}=0
$$

\subsection{Bulk multiplets on the boundary}

In order to couple bulk and boundary multiplets supersymmetrically, we can decompose the boundary values of bulk multiplets into boundary multiplets.

The bulk vector multiplet restricts to a boundary vector multiplet and a real multiplet. The combination

$$
\nabla_{0}=\frac{i}{2}\left\{Q, Q^{\dagger}\right\}
$$

is a singlet under the B-type $\mathcal{N}=2$ supersymmetry. It will couple minimally to charged boundary matter fields according to their gauge charge under the bulk $U(1)$ symmetry.

The story for bulk chiral multiplets depends on the boundary conditions. If $\phi$ does not appear in a boundary superpotential, then:

$$
\begin{aligned}
\left.\nabla_{1} \phi\right|_{\partial \Sigma} & =0 \\
\left.\eta\right|_{\partial \Sigma} & =0 \\
\left.\nabla_{1} \Theta\right|_{\partial \Sigma} & =0 .
\end{aligned}
$$


The supersymmetry variations in Appendix A imply that $\phi$ and $\Theta$ form a boundary chiral multiplet, and $\eta$ and $F$ form a (trivial) boundary fermi multiplet. RG flow may induce a magnetic field coupling for the charged bulk fields at the boundary. This would make them analogous to the $\wp$ multiplet described above. Our considerations will be insensitive to this issue.

If $\phi$ is fixed by a boundary superpotential,

$$
\begin{aligned}
\left.\nabla_{0} \phi\right|_{\partial \Sigma} & =0 \\
\left.\Theta\right|_{\partial \Sigma} & =0 .
\end{aligned}
$$

In this case, the supersymmetry transformations imply that $\eta, F$ form a Fermi multiplet with a deformed chiral constraint $Q^{\dagger} \eta \sim \nabla_{1} \phi$.

\section{A boundary Lagrangian}

Next we write down the complete boundary interactions in an example. Take a Fermi multiplet $\beta$ such that $\bar{D} \beta_{a}=\sqrt{2} \wp E_{a}(\phi)$, with superpotential coupling

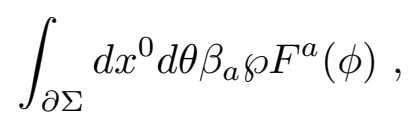

where $\wp$ is a boundary chiral multiplet such that $\bar{D} \wp=0$. Then the kinetic and superpotential terms are:

$$
\begin{aligned}
S_{B}= & \sum_{a} \int d x^{0}\left[i\left(\beta_{a}^{\dagger} \nabla_{0} \beta_{a}-\nabla_{0} \beta_{a}^{\dagger} \beta_{a}\right)+\left|b_{a}\right|^{2}-\left|\wp E_{a}\right|^{2}\right. \\
& +\left(-i \beta_{a}^{\dagger} E_{a} \xi-i \beta_{a}^{\dagger} \wp \partial_{i} E_{a} \Theta^{i}+\text { h.c. }\right) \\
& \left.+\left(i \beta_{a} F^{a} \xi+i \beta_{a} \wp \sum_{i} \partial_{i} F_{a} \Theta^{i}+b_{a} \wp F^{a}+\text { h.c. }\right)\right] .
\end{aligned}
$$


If we integrate out the boundary auxiliary fields, the boundary Lagrangian becomes:

$$
\begin{aligned}
\mathcal{L}_{\partial \Sigma} & =-\sum_{a}\left(2|\wp|^{2}\left|F^{a}\right|^{2}+|\wp|^{2}\left|E_{a}\right|^{2}\right) \\
& +i\left(\beta_{a}^{\dagger} \nabla_{0} \beta_{a}-\nabla_{0} \beta_{a}^{\dagger} \beta_{a}\right)-i\left(\nabla_{0} \wp^{\dagger}\right) \wp+i \wp^{\dagger} \nabla_{0} \wp \\
& +\left(-i \sum_{a} \beta_{a}^{\dagger}\left|E_{a}\right|^{2} \beta_{a}-i \beta_{a}^{\dagger} \wp \partial_{i} E_{a} \Theta^{i}+\text { h.c. }\right)+\left(-\sum_{a} \beta_{a}^{\dagger}\left|F^{a}\right|^{2} \beta_{a}+i \beta_{a} \wp \sum_{i} \partial_{i} F_{a} \Theta^{i}+\text { h.c. }\right) \\
& +\left(i \nabla_{1} p\right)^{\dagger} p+\eta_{p}^{\dagger} \Theta_{p}+\text { h.c. } \\
& +a_{0}\left(j_{s}-1\right) \\
& +\sigma\left(-\partial_{+} \sigma^{\dagger}-\frac{1}{\sqrt{2}}\left(i D-\frac{v_{+-}}{\sqrt{2}}\right)\right)+\sigma^{\dagger}\left(\partial_{-} \sigma+\frac{1}{\sqrt{2}}\left(i D+\frac{v_{+-}}{\sqrt{2}}\right)\right) \\
& +i\left(\lambda_{+}^{\dagger} \lambda_{+}-\lambda_{-}^{\dagger} \lambda_{-}\right)+\frac{1}{\sqrt{2}}\left(\sigma^{\dagger} t^{\dagger}-\sigma t\right)-\frac{i}{4}\left(\sigma-\sigma^{\dagger}\left(\sum_{i} q_{i}\left|\phi_{i}\right|^{2}\right) .\right.
\end{aligned}
$$

The boundary term involving the bulk $p$ field will be explained in $§ 3.7$.

\subsection{Gauging the special symmetry}

As mentioned in $\S 2$, because the massless boundary fermions live in a vector bundle $V$, the Hilbert space on the boundary will transform in a sum of representations of the structure group of $V$. The $\beta$ s create $2^{r}$ states. To find the correct rank- $r$ Hilbert space at one end of the string, we make a projection.

To motivate this, consider $r$ coincident branes in flat space. We can model this by introducing $r$ free boundary fermi multiplets $\beta_{a}$. These fermions acting on the vacuum give $2^{r}=1+r+\left(\begin{array}{c}r \\ 2\end{array}\right)+\cdots$ states. To obtain an $r$-dimensional space of Chan-Paton factors, we project onto states with boundary fermion number one.

Since our boundary fermions interact, boundary fermion number is no longer conserved. However, the boundary fermion number symmetry is replaced by the global boundary symmetry (2.5) mentioned above. We will project onto states which have charge 1 under this symmetry. This is accomplished by including a boundary vector, $a_{0}$, to act as a Lagrange multiplier

$$
\mathcal{L}_{\text {bdy }} \ni a_{0}\left(j_{s}-1\right)
$$

where

$$
j_{s}=: \beta^{\dagger} \beta:-: \wp^{\dagger} \wp:
$$

is the boundary symmetry current. 


\subsection{The Warner problem}

In the presence of a B-type boundary, the bulk worldsheet superpotential is no longer supersymmetric. Its $Q^{\dagger}$ variation is:

$$
\begin{aligned}
Q^{\dagger} \int_{\Sigma} \int d^{2} \theta W & =\int d x^{0} \Theta^{\bar{j}^{\dagger}} \frac{i}{\sqrt{2}}\left(\eta_{i \bar{\jmath}} F^{i}-2 \bar{\partial}_{\bar{\jmath}} W^{\dagger}\right) \\
& \sim \int_{\partial \Sigma} Q W .
\end{aligned}
$$

This does not vanish even if we impose the equations of motion for $F$.

There are many options for dealing with this term. There is a family of boundary terms whose $Q^{\dagger}$ variation cancels the Warner term when the auxiliary fields in the bulk chiral multiplets satisfy their equations of motion. For example for the quintic with

$$
W=p G(\phi)
$$

one can add

$$
\Delta \mathcal{L}_{\mathrm{bdy}}=\int d \theta p \eta^{p \dagger}+\text { h.c. }
$$

which has

$$
Q^{\dagger} \int d \theta p \eta^{p \dagger}=\int d \theta p F^{p \dagger} .
$$

When the auxiliary field $F^{p}$ is evaluated on-shell, this gives

$$
Q^{\dagger} \Delta \mathcal{L}_{\text {bdy }} \sim \int d \theta p G(\phi)+\text { h.c. }
$$

which cancels the Warner variation. This solution works for any quasihomogeneous bulk superpotential.

Another approach is to set $W=0$ as one of the boundary conditions for the bulk chiral multiplets. Then the natural fermionic partner of this condition is precisely $\Theta^{i} \partial_{i} W=$ 0 , and the boundary term (3.41) vanishes. This is natural in that it simply forces the boundaries of the worldsheet to lie in the CY hypersurface. This is equivalent to adding a neutral fermi multiplet $M=\mu+\theta m+\cdots$ such that

$$
\bar{D} M=-\frac{1}{\sqrt{2}}
$$

and writing a term of the form (3.19) with $S=W$. The supersymmetry variation of this action will cancel (3.41). This is the off-shell version of the fix used in [38]. The constraint

(3.45) is the origin of the inhomogenous terms in the supersymmetry transformation in [38].

We believe that both these solutions will lead to sensible conformal theories. 


\subsection{Symmetries of bulk and boundary fields}

\section{Bulk fields}

We will first review the global symmetries of the bulk fields [9]. There are left- and right-moving $\mathrm{R}$-symmetries acting on the bulk chiral fields. Under the right-moving $\mathrm{R}$ symmetry the supersymmetry current $Q^{-}$has charge 15 ; the Grassman variable $\theta^{+}$has charge 1 ; and the fields $\left(\psi_{+}, F, \sigma, \lambda_{-}\right)$have charges $(-1,-1,1,1)$. Under the left-moving R-symmetry, the supersymmetry current $Q^{+}$has charge 1 ; the Grassman variable $\theta^{-}$has charge 1 ; and the fields $\left(\psi_{-}, F, \sigma, \lambda_{+}\right)$have charges $(-1,-1,-1,1)$. These symmetries are non-anomalous so long as the sum of charges of the chiral multiplets vanishes.

The bulk superpotential generically breaks the R-symmetry. However, in absence of the superpotential there was an additional global symmetry under which each superfield $\Phi^{i}$ could be assigned arbitrary charge $k_{i}$. By adding this symmetry to the R-charge, we find new left and right moving $R$ symmetries under which $W$ has left- and right- moving R-charge 1. This symmetry is diagonal; it must be added to both the left- and right-moving R-charges.

For B-type boundary SCFTs, only the sum of the R-charges is preserved:

$$
R_{t o t, i}=R_{+}+R_{-}+2 k_{i}
$$

\section{Boundary fields}

Since bulk and boundary fields are coupled, both will be charged under the conserved R-symmetry. The R-charges of the boundary fields can be deduced from the charges of bulk fields which restrict to the boundary. One will again have to compose symmetries of the boundary theory with the naive R-symmetry to make a symmetry which is respected by the interactions. There is no anomaly in $(0+1)$ dimensions so this symmetry will survive quantization. We will assume that this $U(1)_{R}$ flows to the expected R-symmetry of the infrared $\mathcal{N}=2$ superconformal algebra.

5 The relation between the chiral bulk supercharges $Q^{ \pm}$and the B-type supercharges $Q, Q^{\dagger}$ is described in the Appendices. 


\section{Sheafy variables}

Given a description of a Chan-Paton bundle as the cohomology of an arbitrary sequence of sums of line bundles, a prescription was given in [26] for making a linear sigma model of the type detailed above. However, [26] focused on bundles which have constant fiber dimension over the entire Calabi-Yau manifold.

One linear model for branes of finite codimension, i.e. sheaves with nonconstant fiber dimension over the CY, was described in $§ 2.3$. Simply add a boundary fermi multiplet $\gamma$ of charge $-d \equiv-$ degree $(S(\phi))$ and add a boundary superpotential

$$
\int d \theta \gamma S(\phi) .
$$

This produces a boundary energy $\sim|S(\phi)|^{2}$ which confines the string boundary to the hypersurface. This boundary theory would describe a D-brane localized at $S(\phi)=0$. In this section we present another formulation of this model, which is useful because it makes e.g. the possibility of transitions between Higgs and Coulomb branches in the D-brane moduli space more transparent.

Introduce a boundary fermi multiplet $\beta$ of bulk gauge charge $-d$, and a gauge neutral boundary chiral multiplet $\wp$, and project as usual onto the charge one sector of the boundary symmetry (2.5). Instead of (4.1), add

$$
\int d \theta \wp \beta S(\phi) .
$$

The resulting vacuum equations set $\wp S(\phi)=0$. Away from the locus $\mathcal{S} \equiv\{S=0\}$ this is accomplished by setting $\wp=0 ; \beta$ is massed up by $\xi$. Away from the hypersurface, therefore, there is no way to satisfy the boundary charge projection on the vacuum manifold. When $S=0$, on the other hand, there is no constraint on $\beta$ or $\wp$, but the charge projection picks out a single Chan-Paton state which we will describe in detail below. The resulting D-brane is the sheaf which is the cohomology of the sequence

$$
0 \rightarrow \mathcal{O}(-d) \stackrel{S(\phi)}{\rightarrow} \mathcal{O} \rightarrow 0 .
$$

Away from $\mathcal{S}$ there is no cohomology; over the hypersurface, the map degenerates and a single cohomology element appears at adjacent nodes.

This method of building a brane on a subcycle has also been described from the spacetime point of view, e.g. in [41,42,43]. At large volume, adjacent vector bundles in 
a sequence have opposite-signed D6-brane charge. The map between them represents the open string tachyon. The sequence above describes a D6-brane with D4-brane charge, and an anti-D6 brane; they annihilate to leave a D4-brane behind.

To translate between the two descriptions of hypersurfaces we have given, one can think of $\gamma$ as a boundary-gauge-symmetry-invariant composite of $\wp$ and $\beta$. We refer to the description using $\wp$ and $\beta$ as "sheafy variables" and employ it in the next section when we discuss transitions in open-string moduli space.

The reader may be confused by the fact that in the sheafy description there seem to be two Chan-Paton states localized at the zero set $\mathcal{S}$, one created by $\beta$ and one created by $\wp^{\dagger}$. That this is not the case can be seen by calculating the cohomology of $Q^{\dagger}$ acting on boundary states. As explained in more detail in $\S 6$, this yields the number of massless open string states in the Ramond sector.

Consider an example where there is just a single bulk chiral field $z$ parametrizing a copy of $\mathbb{C}$. Take $\mathcal{S} \equiv\{S(z)=z=0\}$, i.e. a D0-brane at the origin, as described by the sequence (4.3). Finally, introduce an additional space-filling brane with a single trivial Chan-Paton state, i.e. a D2-brane. We will find the massless strings stretching between our brane made by the sequence (4.3) and the D2-brane. Let us start with a vacuum of the interesting end of the string which satisfies

$$
0=\Theta|0\rangle=\beta^{\dagger}|0\rangle=\wp|0\rangle
$$

Imposing the boundary charge projection, an arbitrary state in the Hilbert space is

$$
|\psi\rangle=f_{1}\left(z, z^{\dagger}\right) \beta|0\rangle+f_{2}\left(z, z^{\dagger}\right) \beta \Theta^{\dagger}|0\rangle+f_{3}\left(z, z^{\dagger}\right) \wp^{\dagger}|0\rangle+f_{4}\left(z, z^{\dagger}\right) \wp^{\dagger} \Theta^{\dagger}|0\rangle .
$$

Now we want to solve for $Q^{\dagger}|\psi\rangle=0$ modulo $Q^{\dagger}$-exact states. Reducing to zeromodes, the relevant terms in $Q^{\dagger}$ are

$$
Q^{\dagger}=\Theta^{\dagger} \bar{\partial}+\beta \wp z
$$

Acting on the first term on the RHS of (4.5), we find:

$$
Q^{\dagger} f_{1}(z, \bar{z}) \beta|0\rangle=i \bar{\partial} f_{1} \Theta^{\dagger} \beta|0\rangle
$$

If $f_{1}(z)$ is holomorphic, this state is in the kernel of $Q^{\dagger}$. Furthermore, $Q^{\dagger}$ acts on the third term on the RHS (4.5) to give:

$$
Q^{\dagger} f_{3}(z) \wp^{\dagger}|0\rangle=i z f_{3}(z) \beta|0\rangle
$$


Finally, the second and fourth term on the RHS of (4.5) are Q-exact.

Thus, within cohomology,

$$
f_{1}(z) \simeq f_{1}(z)+z f_{3}(z)
$$

The cohomology representatives live in the ring $\mathbb{C}[z] /\langle z\rangle$, the local ring at $z=0$ (c.f. 444). There is a single cohomology generator localized at $z=0$. This is our state, up to $Q^{\dagger}$ descendants.

We can see even more explicitly that the string endpoint is stuck at the origin and has a single Chan-Paton factor by solving for the ground state wavefunction of the worldsheet zero modes, which is killed by both $Q^{\dagger}$ and $Q$. To do this exactly we need the IR effective action for the zero modes. In this example the bulk theory is a free scalar field theory and the relevant perturbations lie entirely on the boundary. If we assume that the RG flow does not add anything to the bulk kinetic terms (as appears to be the case in related work [28], and if the boundary superpotential satisfies a nonrenormalization theorem similar to that for the bulk superpotential, then the only effect of the RG flow in the presence of the relevant boundary perturbation (4.2) will be to change the anomalous dimension of the superpotential. As the term (4.2) is relevant along the RG flow, it will be dressed with a factor $M^{\alpha}$, where $M \rightarrow \infty$ in the IR and $\alpha$ is positive.

Altering the supercharges accordingly, the ground state should satisfy:

$$
\begin{aligned}
& 0=Q^{\dagger}\left|\psi_{0}\right\rangle=\left(-i \bar{\partial} f_{1}-i M^{\alpha} z f_{4}\right) \Theta^{\dagger} \beta|0\rangle+\left(-i \bar{\partial} f_{3}\right) \Theta^{\dagger} \wp^{\dagger}|0\rangle+M^{\alpha}\left(i z f_{3}\right) \beta|0\rangle \\
& 0=Q\left|\psi_{0}\right\rangle=\left(-i f_{1} \bar{z}-i M^{\alpha} \partial f_{4}\right) \wp^{\dagger}|0\rangle+M^{\alpha}\left(i \partial f_{2}\right) \beta|0\rangle+\left(-i \bar{z} f_{2}\right) \wp^{\dagger} \Theta^{\dagger}|0\rangle
\end{aligned}
$$

where we have ignored time derivatives since $Q^{\dagger}=Q=0$ implies $H=0$. The first equation in (4.10) requires that $f_{3}$ is holomorphic, with support only at $z=0$; thus $f_{3}=0$. Similarly, the second equation leads to $f_{2}=0$. Rotational invariance implies that the lowest-energy state $\left|\psi_{0}\right\rangle$ depends only on $(z \bar{z})$. Therefore,

$$
\left|\psi_{0}\right\rangle \sim A(M) e^{-M^{\alpha}|z|^{2}}\left(\beta|0\rangle+\wp^{\dagger} \Theta^{\dagger}|0\rangle\right)
$$

In the infrared, as $M \rightarrow \infty$, the ground state will become a delta function at $\mathcal{S}=\{z=0\}$. This has also been observed in [45]. 


\subsection{Unions and intersections}

In this section, we explain a number of ways to combine two boundary linear sigma models to make another. For argument, we discuss two transverse hypersurfaces of a CY defined by $S_{1}(\phi)=0$ and $S_{2}(\phi)=0$ with degrees $d_{1}$ and $d_{2}$.

\section{Intersections}

Here are three distinct ways to make the strings end on the intersection $\left\{S_{1}=S_{2}=0\right\}$ :

1. As described in $\S 2$, add a boundary superpotential:

$$
W=\gamma_{1} S_{1}+\gamma_{2} S_{2}
$$

The vacuum energy $\left|S_{1}\right|^{2}+\left|S_{2}\right|^{2}$ only vanishes on the intersection.

2. The tensor product of two sheaves has support on the intersection of the support of the two sheaves. Given the resolution of two sheaves by a sequence of line bundles, there is a formula for such a resolution of the tensor product whose derivation from the LSM is described in Appendix C. In this example, it gives:

$$
\begin{gathered}
0 \rightarrow \mathcal{O} \stackrel{\stackrel{S_{1}}{\longrightarrow} \mathcal{O}\left(d_{1}\right) \rightarrow 0}{ } \bigotimes_{0 \rightarrow \mathcal{O}} \stackrel{\stackrel{S_{2}}{\longrightarrow} \mathcal{O}\left(d_{2}\right) \rightarrow 0}{=} \\
0 \rightarrow \mathcal{O} \stackrel{\left[\begin{array}{l}
S_{1} \\
S_{2}
\end{array}\right]}{\longrightarrow} \mathcal{O}\left(d_{1}\right) \oplus \mathcal{O}\left(d_{2}\right) \stackrel{\left[-S_{2}, S_{1}\right]}{\longrightarrow} \mathcal{O}\left(d_{1}+d_{2}\right) \rightarrow 0 .
\end{gathered}
$$

The field content is a neutral $\wp$, two $\beta$ 's of charges $d_{1,2}$, and a $\tilde{\wp}$ of charge $-\left(d_{1}+d_{2}\right)$ with the constraints on the $Q$ 's and $Q^{\dagger}$ 's determined by the sequence (c.f. [26]).

3. There is another sheafy description of this intersection. Take as boundary fields $\beta_{1,2}$ and $\wp_{1,2}$, coupled via the boundary superpotential:

$$
W=\wp_{1} \beta_{1} S_{1}+\wp_{2} \beta_{2} S_{2}
$$

and project on separate boundary symmetries for each of $\beta_{1}, \wp_{1}$ and $\beta_{2}, \wp_{2}$. Away from the intersection the boundary system cannot satisfy one or the other charge projection while staying on the vacuum manifold. 


\section{Unions}

Three analogous models for the union $\left\{S_{1}=0\right\} \cup\left\{S_{2}=0\right\}$ are:

1. The scheme variables union is simply to use a single boundary fermion of charge $-d_{1}-d_{2}$ and add

$$
W=\gamma S_{1}(\phi) S_{2}(\phi)
$$

The boundary energy is then $\left|S_{1} S_{2}\right|^{2}$ and it vanishes if one lies on either hypersurface. This gives a scheme-theoretic description in the following sense. Notice that if $S_{1}$ and $S_{2}$ are the same, one gets multiple Chan-Paton sectors. In this sense, this boundary linear model keeps track of the fact that a pair of coincident branes is a non-reduced scheme. Related observations were made in [46].

2. As with a single hypersurface, we can describe this in "sheafy" variables. By this we mean replace $\gamma$ by a fermion $\beta$ and a boson $\wp$, gauge the new boundary symmetry, and add

$$
W=\wp \beta S_{1}(\phi) S_{2}(\phi)
$$

3. While tensoring sheaves intersects their supports, adding them gives a sheaf whose support is the union. Simply adding together the sequences we find:

$$
\begin{gathered}
0 \rightarrow \mathcal{O} \stackrel{S_{1}}{\rightarrow} \mathcal{O}\left(d_{1}\right) \rightarrow 0 \\
\bigoplus \\
0 \rightarrow \mathcal{O} \stackrel{S_{2}}{\rightarrow} \mathcal{O}\left(d_{2}\right) \rightarrow 0 \\
= \\
0 \rightarrow \mathcal{O} \oplus \mathcal{O}^{\left[S_{1}, S_{2}\right]} \mathcal{O}\left(d_{1}\right) \oplus \mathcal{O}\left(d_{2}\right) \rightarrow 0
\end{gathered}
$$

The superpotential is the same as (4.14). But if there is just a single charge projection, we obtain the union of the two branes because the string ends on one brane or the other. So this is really the analog of the third way of intersecting - we just do one charge projection instead of two. This is also consistent with the fact that if only one of the boundary symmetries is gauged, then there is one left over which acts as the second $U(1)$ spacetime gauge symmetry - the branes can move independently. 


\section{Applications and consequences}

\subsection{Monodromy in closed-string moduli space}

The open string linear sigma model is an ideal tool for thinking about transport of branes in closed string moduli space. Choose some boundary field content to make a particular D-brane in the IR. If we move along a closed path in Kähler moduli space, we come back to the same bulk LSM, but with possibly different boundary data. This determines an action of the monodromy group on the branes themselves, and not just on their charges. Such a refinement of the action of the monodromy group on branes has also been observed in the approach of [41,47,42] where branes are considered as objects in the derived category.

In the case of the quintic, there are three monodromy generators [48]. At large volume, the imaginary part of the complexified Kähler form - represented in the linear model by the worldsheet theta angle - has periodicity $2 \pi$. A shift by $2 \pi$ at large $r$ is a noncontractible loop and should generate the "large-volume monodromy." A loop about $r=0$ should generate a monodromy which corresponds to the monodromy about the conifold point in the mirror CY. Finally, at large negative $r$ a shift of $\theta$ by $2 \pi$ is also a noncontractible loop in the closed string GLSM. Since $r \rightarrow-\infty$ describes the Gepner point which is a $\mathbb{Z}_{5}$ orbifold point in the moduli space, this shift of $\theta$ should generate the $\mathbb{Z}_{5}$ monodromy action about the Gepner point.

\section{Large radius}

Consider a single D6-brane on the quintic, modeled by a single neutral boundary fermion which carries charge 1 under the gauged boundary symmetry. The pertinent terms in the boundary action are

$$
\mathcal{L}_{b d y} \ni\left(\frac{\theta}{2 \pi} v_{0}+j v_{0}+\left(j_{s}-1\right) a_{0}\right)
$$

where $v_{0}$ is the bulk worldsheet gauge field, $j$ is the bulk gauge current, $j_{s}$ is the boundary symmetry current, and $a_{0}$ is the boundary vector field in (3.39).

Now, shifting $\theta \rightarrow \theta+2 \pi \alpha$ adds to this

$$
\delta \mathcal{L}=\alpha v_{0}
$$


(this is only gauge invariant if $\alpha$ is an integer multiple of $2 \pi$ ). This is equivalent to shifting the bulk gauge charges of all fields by their boundary charge:

$$
j \rightarrow j+\alpha j_{s} .
$$

In our example, this means that when $\alpha=1$, we can remove the term (5.2) by giving the boundary fermion bulk gauge charge 1. But this shifts the chern classes of the bundle; in particular, it adds one unit of four-brane charge. This is the expected large-radius monodromy.

This is the right answer for any boundary field content. Adding the boundary symmetry generator to the gauge charge has the effect of tensoring the bundle with the line bundle $\mathcal{O}(1)$ over the projective space. This is precisely the effect of moving the NS B-field through one period [48,5].

\section{Other monodromy generators}

An understanding of the other independent monodromy generator requires an analysis of the effective theory at the conifold singularity. Work is in progress in this direction. Here we restrict ourselves to a few suggestive observations.

Firstly, even at large negative $r, \theta \rightarrow \theta+2 \pi$ acts by adding the boundary current to the gauge current. So it seems that the idempotence of the LG monodromy action is related to the $\mathbb{Z}_{5}$-valuedness of the worldsheet gauge charge.

Secondly, it generates the expected monodromy on the branes following the discussion in [20]. A "fractional brane" in their model corresponds to a brane whose Chan-Paton factors have $\mathbb{Z}_{5}$ charge under the orbifold group, corresponding to the different irreducible representations of $\mathbb{Z}_{5}$. The quantum symmetry at the orbifold point rotates these irreps and so shifts all of the charges by 1 mod 5 .

Finally, the trivial representation is believed to correspond to the D6-brane at the Gepner point [20]. This is the same description as at large radius. The fact that the D6-brane has no monodromy about $r=0$ suggests that we have the right description at the Gepner point. 


\subsection{Degenerations and Singularities}

Singularities in the CFT moduli space are especially important. They provide a window into nonperturbative physics, and can give rise to the singularities of the spacetime superpotential which are expected in $\mathcal{N}=1$ supergravity [49,13. In the closed string GLSM, singularities of the CFT appear at points in the GLSM moduli space where the vacuum manifold becomes noncompact [9]. This should also hold true in the open string case. Furthermore, apparent singularities in the open string moduli space, such as "small instanton" singularites, signal enhanced gauge symmetries or the existence of a branch structure in the moduli space.

Singularities in the closed string moduli space

For closed strings, singularities in the complex structure moduli space occur when $G$ is not transverse; then, as in [9], there is a branch where $p$ and some of the $\phi$ s diverge with zero energy cost. If there are boundary terms of the form (3.19), where $S$ depends only on the bulk fields, $\phi^{i}$ will not always be able to diverge consistent with $S^{A}(\phi)=0$, so the $D$-term keeps $p$ from diverging. The boundary CFT will not be singular (i.e. the D-brane physics will be smooth) at many points where the closed string CFT would be singular.

If $S=0$ is consistent with the nonzero $\phi^{i}$ necessary for the existence of the noncompact $p$ branch, then the full boundary CFT will be singular. In other words, there can be singularities in the relevant boundary CFT as well as the closed string CFT when the D-brane intersects the closed string singularity.

Singularities in the Kähler moduli space occur when $\sigma$ has a noncompact branch. For the closed GLSM on the quintic, this occurs at $r=\theta=0$. It is an interesting question whether D-brane physics is singular at this point - see for example [3]. If the Chan-Paton factors for a given state have nontrivial gauge charge then the answer is uncertain. When $\sigma$ is large all of the charged fields have masses of order $|\sigma|^{2}$. The charged boundary fermions create a constant electric field in the bulk. Competing effects exist: on the one hand one expects screening of the bulk electric field via Schwinger pair production, on the other hand the mass of the bulk charged fields (which must be pair-produced to provide the screening) grows quickly down the $\sigma$ branch. It would be interesting to disentangle the physics of the potential singularity by performing a delicate analysis of the IR limit (as was done for closed strings in [13]).

Singularities from boundary fields? 
If the boundary field $\wp$ has no potential, one might worry that a noncompact branch develops and the CFT is singular. For example, in the Calabi-Yau phase, $\wp$ is usually frozen to zero by the term $\sum_{a}|\wp|^{2}\left|F^{a}\right|^{2}$, which gives $\wp$ a large mass since the $F^{a}$ are generically nonvanishing at $S=0$ in the Calabi-Yau. However, one could choose a bundle with singular points, where the $F^{a}$ all vanish on $S=0$. Even more uncomfortably, in the Landau-Ginzburg phase $\phi=F=0$. It would be bizarre if the CFT was singular in an open set in the moduli space.

However a $\wp$ branch does not exist. The boundary charge projection gives a finite number of states in the Hilbert space of boundary fields. The $\wp$ field is a "quantum dimension" in the target space, and cannot cause divergences in the path integral. The integral over $a_{0}$ projects onto:

$$
j_{s}=\sum_{a}: \beta_{a}^{\dagger} \beta_{a}:-: \wp^{\dagger} \wp:=1
$$

Since the $\beta$ s are fermionic, the positive contribution in (5.4) is bounded; therefore (when (5.4) can be satisfied), $\wp$ contributes a finite volume factor to the path integral and does not give any new branches to the path integral.

\section{Enhanced gauge symmetries}

Despite the absence of a $\wp$ branch, there is significant physics when a bundle degenerates. If a locus $\mathcal{D}$ exists such that for $\phi_{\star}^{i} \in \mathcal{D}, F^{a}\left(\phi_{\star}\right)=G\left(\phi_{\star}\right)=0$, then in addition to the bundle $V$, we get a sheafy variables description of a lower-dimensional brane - namely a sheaf with support over $\mathcal{D}$. The small instanton limit [50 of the D0-D4 system is the classic example of such a degeneration.

In addition, we can sometimes tune parameters such that additional global symmetries arise, in addition to (2.5) which rotates all $\beta$ 's and $\wp$ 's oppositely. Then additional spacetime gauge symmetries should appear.

\section{Small Instanton "Singularities"}

The "sheafy variables" construction of D-branes allows us to easily study transitions between branches of D-brane moduli spaces. First we will study two D2-branes filling orthogonal complex lines in a flat $\mathbb{C}^{3}$. The "Higgs branch" can occur when they lie in a common $\mathbb{C}^{2}$ and can be deformed into a single D2-brane; the "Coulomb branch" occurs when they are separated along the complex line orthogonal to both of them. If we replaced 
$\mathbb{C}^{3}$ by $T^{6}$ this would be the T-dual of the small instanton singularity. We will then proceed to a direct study of small instantons in the D0-D4 system.

Consider the linear model which flows to the Coulomb branch. We take as boundary fields $\beta, \tilde{\beta}, \wp_{1,2}, \beta^{\prime}, \tilde{\beta}^{\prime}$. We wish to describe a background with one two-brane located at $z_{1}=0, z_{3}=0$, and the other at $z_{2}=0, z_{3}=a$.

Using the "sheafy variables" description, the complex defining this configuration is

$$
\begin{aligned}
& \left(\begin{array}{c}
0 \rightarrow \mathcal{O} \stackrel{z_{1}}{\rightarrow} \mathcal{O} \rightarrow 0 \\
\otimes \\
z_{3} \\
0 \rightarrow \mathcal{O} \rightarrow \mathcal{O} \rightarrow 0
\end{array}\right) \bigoplus\left(\begin{array}{c}
0 \rightarrow \mathcal{O} \rightarrow \mathcal{O} \rightarrow 0 \\
\otimes \\
z_{3}-a \\
0 \rightarrow \mathcal{O} \rightarrow \mathcal{O} \rightarrow 0
\end{array}\right) \\
& =
\end{aligned}
$$

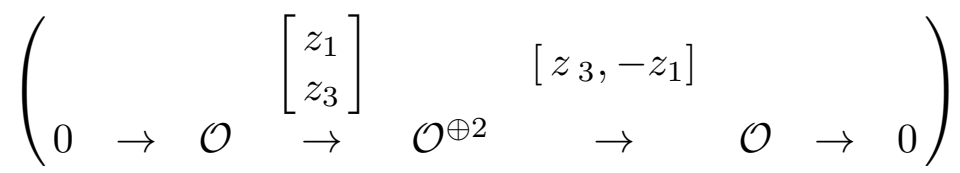

$$
\begin{aligned}
& \bigoplus
\end{aligned}
$$

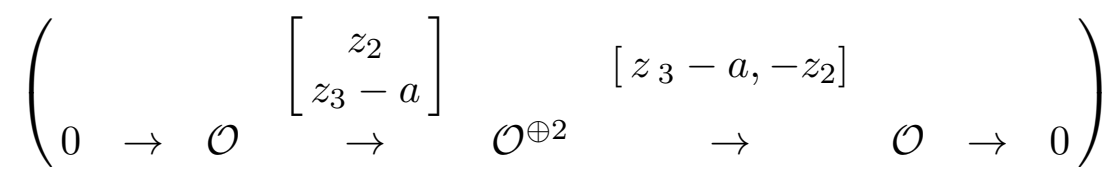

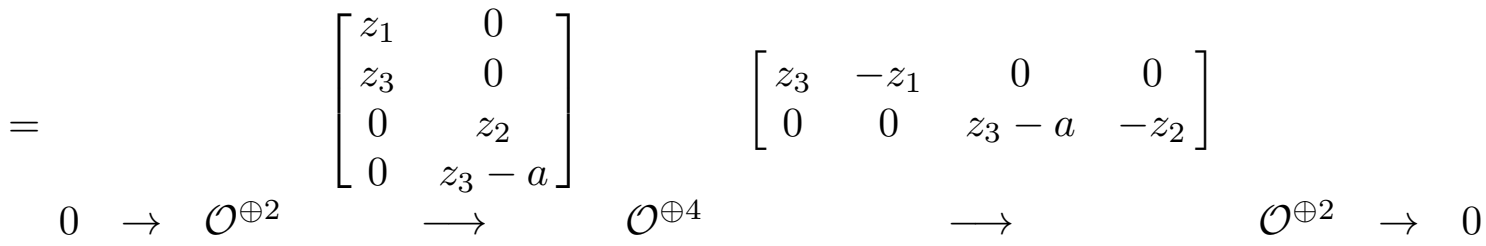

In other words, the deformed chiral constraints are

$$
\begin{array}{lccc}
Q^{\dagger} \beta=0 & Q^{\dagger} \wp_{1}^{\dagger}=z_{1} \beta & Q^{\dagger} \wp_{2}^{\dagger}=z_{3} \beta & Q^{\dagger} \beta^{\prime}=z_{3} \wp_{1}^{\dagger}-z_{1} \wp_{2}^{\dagger} \\
Q^{\dagger} \tilde{\beta}=0 & Q^{\dagger} \tilde{\wp}_{1}^{\dagger}=z_{2} \tilde{\beta} & Q^{\dagger} \tilde{\wp}_{2}^{\dagger}=\left(z_{3}-a\right) \tilde{\beta} & Q^{\dagger} \tilde{\beta}^{\prime}=\left(z_{3}-a\right) \tilde{\wp}_{1}^{\dagger}-z_{2} \tilde{\wp}_{2}^{\dagger}
\end{array}
$$

and the on-shell supersymmetry transformations are

$$
\begin{array}{cccc}
Q^{\dagger} \beta^{\dagger}=-z_{1} \wp_{1}-z_{3} \wp_{2} & Q^{\dagger} \wp_{1}=-z_{3}{\beta^{\prime}}^{\dagger} & Q^{\dagger} \wp_{2}=z_{1}{\beta^{\prime}}^{\dagger} & Q^{\dagger}{\beta^{\prime \prime}}^{\dagger}=0 \\
Q^{\dagger} \tilde{\beta}^{\dagger}=-z_{2} \tilde{\wp}_{1}-\left(z_{3}-a\right) \tilde{\wp}_{2} & Q^{\dagger} \tilde{\wp}_{1}=-\left(z_{3}-a\right) \tilde{\beta}^{\prime \dagger} & Q^{\dagger} \tilde{\wp}_{2}=z_{2} \tilde{\beta}^{\prime \dagger} & Q^{\dagger} \tilde{\beta}^{\prime \dagger}=0
\end{array}
$$

One can search for marginal deformations of the system in one of two ways. Either one can look for candidate superpotential terms annihilated by $Q^{\dagger}$ (using the unperturbed EOM and values for the auxiliary fields); or one can look for consistent perturbations of 
the off-shell deformed chiral constraints and add no explicit superpotential term. The two approaches yield equivalent results; here we will take the latter.

We are interested in deformations which preserve the orientation of the branes and more generally the structure of the configuration at infinity, so we only allow deformations of the maps of the complex which are constant independent of $z$. Deformations linear in $z$ would generally alter the orientation of the branes, and higher-order polynomials would cause even more drastic changes in the brane geometry.

For $a \neq 0$ there are no constant deformations of the system other than the obvious ones corresponding to moving the twobrane moduli around on the Coulomb branch.

When $a$ vanishes, however, one can find other consistent perturbations of the deformed chiral constraints, or equivalently deformations of the complex preserving nilpotence of the differential $Q^{\dagger}$.

Specifically, for vanishing $a$ the most general set of constants one can add to the maps of the complex is:

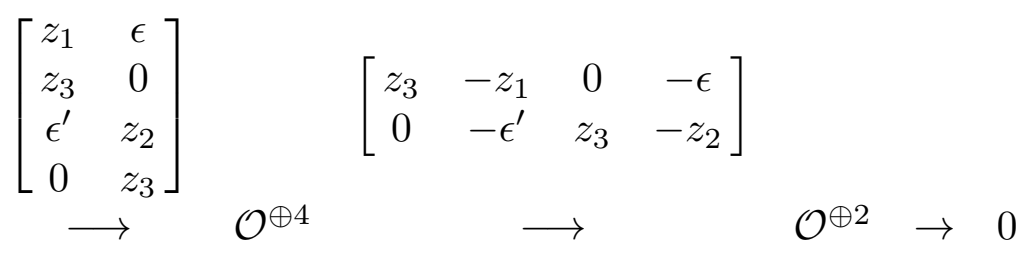

We will now show that for $a=0$ the effect of this perturbation will be to merge the two branes into a single brane covering the locus $z_{3}=0, z_{1} z_{2}=\epsilon \epsilon^{\prime}$. After adding this deformation of the complex, the deformed chiral constraints become

$$
\begin{array}{llll}
Q^{\dagger} \beta=0 & Q^{\dagger} \wp_{1}^{\dagger}=z_{1} \beta+\epsilon_{1} \tilde{\beta} & Q^{\dagger} \wp_{2}^{\dagger}=z_{3} \beta & Q^{\dagger} \beta^{\prime}=z_{3} \wp_{1}^{\dagger}-z_{1} \wp_{2}^{\dagger}-\epsilon \tilde{\wp}_{2}^{\dagger} \\
Q^{\dagger} \tilde{\beta}=0 & Q^{\dagger} \tilde{\wp}_{1}^{\dagger}=z_{2} \tilde{\beta}+\epsilon^{\prime} \beta & Q^{\dagger} \tilde{\wp}_{2}^{\dagger}=z_{3} \tilde{\beta} & Q^{\dagger} \tilde{\beta}^{\prime}=z_{3} \tilde{\wp}_{1}^{\dagger}-z_{2} \tilde{\wp}_{2}^{\dagger}-\epsilon^{\prime} \wp_{2}^{\dagger}
\end{array}
$$

and the on-shell supersymmetry transformations are

$$
\begin{array}{llll}
Q^{\dagger} \beta^{\dagger}=-z_{1} \wp_{1}-z_{3} \wp_{2}-\epsilon^{\prime} \tilde{\wp}_{1} & Q^{\dagger} \wp_{1}=-z_{3} \beta^{\prime \dagger} & Q^{\dagger} \wp_{2}=z_{1} \beta^{\prime \dagger}+\epsilon^{\prime} \tilde{\beta}^{\prime \dagger} & Q^{\dagger} \beta^{\prime \dagger}=0 \\
Q^{\dagger} \tilde{\beta}^{\dagger}=-z_{2} \tilde{\wp}_{1}-z_{3} \tilde{\wp}_{2}-\epsilon \wp_{1} & Q^{\dagger} \tilde{\wp}_{1}=-z_{3} \tilde{\beta}^{\prime \dagger} & Q^{\dagger} \tilde{\wp}_{2}=z_{2} \tilde{\beta}^{\prime \dagger}+\epsilon \beta^{\prime \dagger} & Q^{\dagger} \tilde{\beta}^{\prime \dagger}=0
\end{array}
$$

The condition for the $Q$ and $Q^{\dagger}$ variations of all $\wp$ fields to vanish is

$$
\begin{aligned}
z_{3}\left(\beta, \tilde{\beta}, \beta^{\prime \dagger}, \tilde{\beta}^{\prime \dagger}\right) & =0 \\
\left(z_{1} z_{2}-\epsilon \epsilon^{\prime}\right)\left(\beta, \tilde{\beta}, \beta^{\prime \dagger}, \tilde{\beta}^{\prime \dagger}\right) & =0 .
\end{aligned}
$$

So we see that this deformation moves the support of the brane to the irreducible variety defined by

$$
z_{3}=0, \quad z_{1} z_{2}=\epsilon \epsilon^{\prime}
$$


which represents the Higgs branch of this D2-D2 system.

There is a shortcut to finding the locus of the twobrane. If one takes a generic point in the base space, the matrices defining the complex are all of full rank. Their rank is reduced, leading to nonzero cohomology, exactly when all two by two subdeterminants vanish. This occurs at the locus $z_{3}=0, z_{1} z_{2}=\epsilon \epsilon^{\prime}$.

Higgs-Coulomb transition in the D0-D4 system

The merging of two $D 2$-branes is $T$-dual to the Coulomb-Higgs transition in the 0-4 system. We can also describe this transition directly.

A linear sigma model for a zerobrane separated from a pair of fourbranes is

$$
0 \rightarrow \mathcal{O} \stackrel{d_{1}}{\rightarrow} \mathcal{O}^{\oplus 5} \stackrel{d_{2}}{\rightarrow} \mathcal{O}^{\oplus 5} \stackrel{d_{3}}{\rightarrow} \mathcal{O} \rightarrow 0
$$

with

$$
\begin{gathered}
d_{1} \equiv\left[\begin{array}{c}
z_{1} \\
z_{2} \\
z_{3}-L \\
0 \\
0
\end{array}\right] \\
d_{2} \equiv\left[\begin{array}{ccccc}
0 & z_{3}-L & -z_{2} & 0 & 0 \\
L-z_{3} & 0 & z_{1} & 0 & 0 \\
z_{2} & -z_{1} & 0 & 0 & 0 \\
0 & 0 & 0 & z_{3} & 0 \\
0 & 0 & 0 & 0 & z_{3}
\end{array}\right] \\
d_{3} \equiv\left[\begin{array}{lllll}
z_{1}, & z_{2}, & z_{3}-L & 0, & 0
\end{array}\right] .
\end{gathered}
$$

For generic $L$ the only marginal operators one can add simply correspond to shifts in the positions of the branes. However for $L=0$ one can deform the complex to:

$$
\begin{aligned}
d_{1} \equiv\left[\begin{array}{c}
z_{1} \\
z_{2} \\
z_{3} \\
-B_{1} \\
-B_{2}
\end{array}\right] \\
d_{2} \equiv\left[\begin{array}{ccccc}
0 & z_{3} & -z_{2} & 0 & 0 \\
-z_{3} & 0 & z_{1} & 0 & 0 \\
z_{2} & -z_{1} & 0 & C_{1} & C_{2} \\
0 & 0 & B_{1} & z_{3}+E_{11} & E_{12} \\
0 & 0 & B_{2} & E_{21} & z_{3}+E_{22}
\end{array}\right] \\
d_{3} \equiv\left[\begin{array}{lllll}
z_{1}, & z_{2}, & z_{3}, & -C_{1}, & -C_{2}
\end{array}\right]
\end{aligned}
$$


for $B, C, E$ which satisfy

$$
\left[\begin{array}{ll}
C_{1}, & C_{2}
\end{array}\right]\left[\begin{array}{l}
B_{1} \\
B_{2}
\end{array}\right]=\left[\begin{array}{ll}
E_{11} & E_{12} \\
E_{21} & E_{22}
\end{array}\right]\left[\begin{array}{l}
B_{1} \\
B_{2}
\end{array}\right]=\left[\begin{array}{ll}
C_{1}, & C_{2}
\end{array}\right]\left[\begin{array}{ll}
E_{11} & E_{12} \\
E_{21} & E_{22}
\end{array}\right]=0
$$

The general solution to these constraints consists of a unit doublet $\left(u_{1}, u_{2}\right)$ and three complex numbers $b, c, e$ :

$$
\left[\begin{array}{l}
B_{1} \\
B_{2}
\end{array}\right]=b\left[\begin{array}{l}
u_{1} \\
u_{2}
\end{array}\right] ; \quad\left[\begin{array}{ll}
C_{1}, & C_{2}
\end{array}\right]=c\left[u_{2}, \quad-u_{1}\right] ; \quad\left[\begin{array}{cc}
E_{11} & E_{12} \\
E_{21} & E_{22}
\end{array}\right]=e\left[\begin{array}{cc}
u_{1} u_{2} & -u_{1}^{2} \\
u_{2}^{2} & -u_{1} u_{2}
\end{array}\right]
$$

We will now assume generic values of $b, c, e, u$. There is never cohomology at the first or last node; neither $d_{1}$ nor $d_{3}$ ever has a kernel. $d_{2}$ always has determinant zero, which is to be expected since $d_{1}$ has one-dimensional image, so $d_{2}$ must have at least one-dimensional kernel.

There will be cohomology at the second and third nodes only when the dimension of the kernel of $d_{2}$ is two or higher, the criterion for which is the vanishing of all twenty-five 4 by 4 subdeterminants of $d_{2}$. We find that the matrix of subdeterminants is

$$
z_{3}^{2} \cdot\left[\begin{array}{ccccc}
-b c u_{1} u_{2}, & b c u_{1}^{2}, & c u_{1} z_{3}, & -c u_{1} z_{2}, & c u_{1} z_{1} \\
-b c u_{2}^{2}, & b c u_{1} u_{2}, & c u_{2} z_{3}, & -c u_{2} z_{2} & c u_{2} z_{1} \\
-b u_{2} z_{3} & b u_{1} z_{3} & z_{3}^{2} & -z_{2} z_{3} & z_{1} z_{3} \\
b u_{2} z_{2} & -b u_{1} z_{2} & -z_{2} z_{3} & z_{2}^{2} & -z_{1} z_{2} \\
-b u_{2} z_{1} & b u_{1} z_{1} & z_{1} z_{3} & -z_{1} z_{2} & z_{1}^{2}
\end{array}\right]
$$

Specifically, it factorizes as $z_{3}^{2}$ times a matrix which never vanishes. (The entry in the upper left hand corner, for instance, is constant and nonzero). This means that there are two fibers along the locus $z_{3}=0$ (rather than a single fiber, since the zero is doubled), and no extra fibers at special subloci of $z_{3}=0$. The interpretation is that we have dissolved a zerobrane into a smooth instanton field in the pair of fourbranes. (Note that the value of $e$ drops out of the subdeterminants and we can set it to zero in what follows.)

We can see this even more directly by examining the cohomology restricted to $z_{3}=0$. Setting $e=0$ and computing the kernel of $d_{2}$ we have

$$
d_{2} \equiv\left[\begin{array}{ccccc}
0 & 0 & -z_{2} & 0 & 0 \\
0 & 0 & z_{1} & 0 & 0 \\
z_{2} & -z_{1} & 0 & C_{1} & C_{2} \\
0 & 0 & B_{1} & 0 & 0 \\
0 & 0 & B_{2} & 0 & 0
\end{array}\right]\left[\begin{array}{l}
\beta_{1} \\
\beta_{2} \\
\beta_{3} \\
\beta_{4} \\
\beta_{5}
\end{array}\right]=0
$$




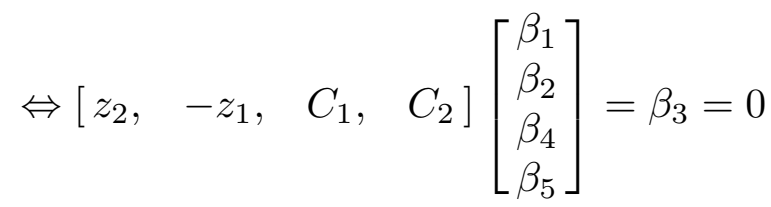

Furthermore, the massless $\beta$ 's are in the kernel of $d_{1}^{\dagger}$, restricted to $z_{3}=0$. That is,

$$
\left[z_{1}^{*}, \quad z_{2}^{*}, \quad B_{1}^{*}, \quad B_{2}^{*}\right]\left[\begin{array}{c}
\beta_{1} \\
\beta_{2} \\
\beta_{4} \\
\beta_{5}
\end{array}\right]=0
$$

In other words, the vector $\left[\beta_{1}, \beta_{2}, \beta_{4}, \beta_{5}\right]$ is a harmonic representative of the cohomology of the complex

$$
\begin{aligned}
& {\left[\begin{array}{c}
z_{1} \\
z_{2} \\
B_{1} \\
B_{2}
\end{array}\right] \quad\left[z_{2},-z_{1}, C_{1}, \quad C_{2}\right]} \\
& 0 \rightarrow \mathcal{O} \rightarrow \mathcal{O}^{\oplus 4} \quad \rightarrow \quad \rightarrow \quad \text { O } \rightarrow 0
\end{aligned}
$$

with

$$
\left[\begin{array}{cc}
C_{1}, & C_{2}
\end{array}\right]\left[\begin{array}{l}
B_{1} \\
B_{2}
\end{array}\right]=0
$$

But the ADHM construction of a single $U(2)$ instanton in $\mathbb{R}^{4}$ works precisely by defining a holomorphic bundle as the cohomology of this same complex. So we have directly demonstrated the ability of a D0 brane to dissolve into D4 branes by open string worldsheet arguments, without any reference to the brane worldvolume gauge theory.

This whole computation should generalize without undue complication to the process of dissolving $k$ instantons in $N$ fourbranes to make a smooth $k$-instanton field in a $U(N)$ gauge theory on $\mathbb{R}^{4}$.

\subsection{Marginal stability transitions: A local worldsheet model}

While many of the quantities which are reliably computed in the open string LSM are independent of the worldsheet FI parameter, it is clear that important aspects of the physics of B-type D-branes do depend on the Kähler moduli. The most striking example is marginal stability transitions, which occur at special loci in the Kähler moduli space. Determining when a transition occurs for a given set of brane charges is a delicate problem which has come under intense recent investigation (see e.g. [5, 6, [7,41]). Since the topological B-model is insensitive to the Kähler parameters, the occurence of such transitions is not 
transparent in the linear model. However, we can understand the local physics of these transitions in the linear model in a simple way. The local model we present below is a good candidate to describe the generic worldsheet behavior in the vicinity of such a transition.

A local model for the spacetime physics of a D-brane undergoing a marginal stability transition is the Fayet model [51]. This is easily seen in the A-model [4,52], where the physics can be reduced to a problem involving intersecting branes.6 The Fayet model is a four-dimensional $\mathcal{N}=1$ field theory with a $U(1)$ gauge symmetry and a single charged field. (In the brane system, there is also a center of mass vector field gauging a $U(1)$ under which all fields are neutral.) The D-term takes the form

$$
D=|\phi|^{2}-\xi
$$

When the FI parameter $\xi$ is positive, there is a supersymmetric vacuum at nonzero $\phi$; the gauge symmetry is broken by this vev and there is a mass gap. For $\xi<0$, there is no way to make $D$ vanish; supersymmetry is broken, and the gauge symmetry is preserved. Classically, the gauge multiplet $\left(A_{\mu}, \lambda_{\alpha}\right)$, and the fermion partner $\psi_{\alpha}$ of $\phi$ are all massless, while $\phi$ is massive. In realizations of this transition in B-type brane systems, $\xi$ is to be identified with a nontrivial function of the Kähler parameter $t=\frac{\theta}{2 \pi}+i r$. There is a supersymmetric brane for $\xi>0$ and the brane decays on the locus $\xi\left(t, t^{\dagger}\right)=0$ in Kähler moduli space.

This simplest model of the spacetime physics is reproduced by the simplest possibility in the LSM. Add a brane-antibrane pair to the linear model - i.e. add a boundary chiral multiplet $\wp$ and a boundary fermi multiplet $\beta$.

To model the behavior when $\xi$ is small and positive, add the operator

$$
f(\xi) \int d \theta \wp \beta
$$

to the boundary action ( $f$ is some nontrivial function of $\xi$, which vanishes when $\xi=0$ ). The $Q^{\dagger}$ complex then takes the form

$$
0 \rightarrow\left\{\beta^{\dagger} \wp^{\dagger}\right\} \rightarrow\left\{\begin{array}{c}
\wp^{\dagger} \wp \\
\beta^{\dagger} \beta
\end{array}\right\} \rightarrow\{\beta \wp\} \rightarrow 0 .
$$

At generic points when $\xi>0, f$ is non-vanishing. The last map from R-charge 0 to $\mathrm{R}$ charge 1 is then onto, and there is no cohomology beyond the center of mass vector at

6 In the B-model, these transitions are related to a chamber structure in the moduli space of stable bundles [53]. 
R-charge 0 . We have a supersymmetric vacuum with the mass gap (for modes other than the decoupled $U(1))$ that we expect. The R-symmetry of the CFT to which we flow must preserve (5.27), so the operator $\beta \wp$ has unit R-charge.

Now consider the behavior at $\xi=0$. Since $f(\xi)=0$ at this point, the maps in (5.28) degenerate and we get a cohomology generator at R-charge zero (in addition to the centerof-mass gaugino $\left.\mathbf{1}=\beta^{\dagger} \beta-\wp^{\dagger} \wp\right)$ which we identify as a new gaugino. Its image under spectral flow is a new massless vector. There is also new cohomology at R-charge 1 of the form $\beta \wp$ which we identify with the vertex operator for $\psi_{\alpha}$, the fermi component of the charged chiral multiplet. The R-charge of this operator is still unity, and so spectral flow generates from it the vertex operator for massless scalar $\phi$. One can show, using the equation

$$
\left[\beta^{\dagger} \beta, \wp \beta\right]=-\wp \beta,
$$

that the string created by this vertex operator indeed carries unit charge under the new vector field, as expected.

Now, what happens as one moves past the spacetime transition point, to the $\xi<0$ region of parameter space of the Fayet model? At $\xi=0$, the model enjoys an extra unbroken $U(1)$ global symmetry - one can rotate $\beta$ and $\wp$ independently. There is no longer a unique candidate for the $U(1)_{R}$ symmetry which appears in the IR $\mathcal{N}=2$ superconformal algebra. We can hypothesize that when $\xi$ is made slightly negative, the R-charge is a linear combination of the two $U(1)$ s under which $\beta \wp$ has charge $>1$. We then find that the scalar $\phi$ obtains a tree-level mass since the conformal weight of the image of $V_{\psi}$ under spectral flow will be different from 1 . The operator $\beta \wp$ is still in the $Q^{\dagger}$ cohomology, so $\psi_{\alpha}$ remains massless.

This scenario will be realized in the following situation. When $\xi>0$, the $\phi$ field has two gauge-inequivalent vacua (the true vacuum, and the tachyonic vacuum at $\langle\phi\rangle=0$ ). Associated with these two vacua are two different boundary CFTs. In the CFT of the supersymmetric vacuum, where the tachyonic perturbation, $\int d \theta \beta \wp$, has been turned on, the conserved R-charge must be such that $\beta \wp$ has unit R-charge. 


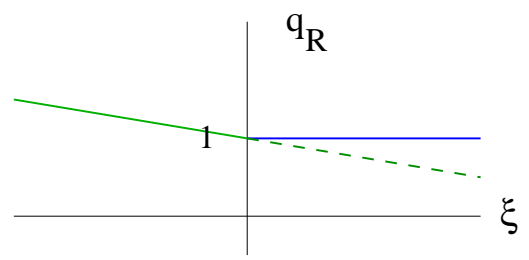

Fig. 1: The R-charge, $q_{R}$, of the operator $\wp \beta$ varies with the spacetime FI coefficient $\xi$. The dashed line indicates the conserved R-charge in the CFT of the tachyonic vacuum at $\langle\phi\rangle=0$.

In the unperturbed CFT of the tachyonic $\langle\phi\rangle=0$ vacuum, there is an extra global boundary $U(1)$ symmetry under which (if necessary by adding to it a multiple of the gauged boundary current) only $\beta$ is charged. Therefore the conserved R-charge in the IR theory can be some linear combination of the bulk R-current and the $\beta$ number current with $r$-dependent (and hence $\xi$-dependent) coefficients determined by the boundary RG flow. It is this gauge-symmetric vacuum (and hence this CFT) which describes the brane at $\xi<0$, and so the R-charge of $\wp \beta$ can vary with $\xi$ when $\xi$ becomes negative.

It would be very interesting to understand in detail, from a microscopic point of view, the appearance of this local model in various D-brane decay processes. It is quite plausible that not only generic marginal stability transitions, but also generic variations of the worldvolume spectrum on a given D-brane, can be accomplished through the judicious addition of brane/anti-brane pairs in this manner.

\section{Massless worldvolume fields}

\subsection{Generalities}

In this section we compute the massless worldvolume spectrum in a particular example. Our linear model flows to the worldsheet-supersymmetric Ramond sector of strings ending on these branes, and it is the states in this sector which are annihilated by $L_{0}$ that we determine. As in [41,42, 13] it should be understood that we are looking at branes in the topological B-model, and the spectrum of strings we are computing corresponds to the massless open (fermionic) strings stretching between such branes. The relation of these "topological" branes to physical branes is not always straightforward: the supersymmetric branes in the topological model satisfy the physical F-flatness conditions but not necessarily the physical D-flatness conditions. The question of which topological branes satisfy the latter condition is equivalent to understanding marginal stability, and loci of marginal 
stability are not manifest in the linear model (and indeed still need to be determined on a more or less case by case basis). In some special cases, e.g. half-supersymmetric D-branes on $K 3$, the enhanced supersymmetry makes it easier to infer properties of the physical branes from the topological model, so after some generalities we will specialize to an example involving branes on $K 3$.

To find the massless open string states, we can study the supersymmetric ground states in the Ramond sector. In this sector, the two unbroken supercharges $Q$ and $Q^{\dagger}$ anti-commute to the Ramond-sector Hamiltonian:

$$
\left\{Q, Q^{\dagger}\right\}=2 L_{0}
$$

By (6.1) and standard results in Hodge theory, we can find the supersymmetric ground states by computing the $Q^{\dagger}$ cohomology. Since the FI terms are $Q$ exact, we can ignore their effects in this computation.

We will find it convenient to compute the cohomology of $Q^{\dagger}$ acting on operators.

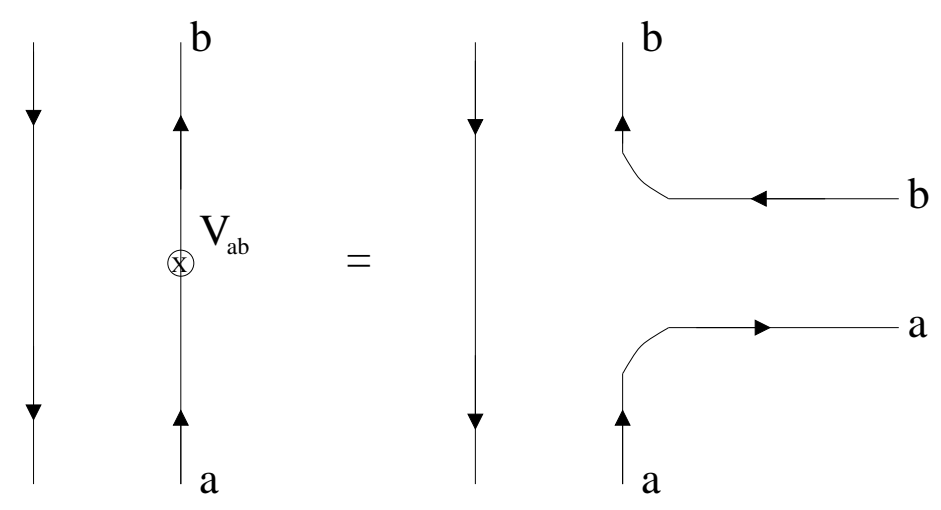

Fig. 2: In the conformal limit, emission of a string stretched between branes is the same as insertion of a boundary-condition-changing vertex operator, $V_{a b} \sim \beta_{b}^{\dagger} \beta_{a}$.

Since, as illustrated in fig. 2, there is a 1-1 correspondence between the open string states and associated boundary condition changing operators, this does not constitute a loss of generality. 


\subsection{Example: A bundle on K3}

Our example consists of three D4-branes

\begin{tabular}{|c|c|c|c|}
\multicolumn{1}{c}{ Field } & \multicolumn{1}{c}{$q_{G}$} & \multicolumn{1}{c}{$q_{R}$} & \multicolumn{1}{c}{$q_{S}$} \\
\hline$\phi^{i}$ & 1 & $\frac{1}{2}$ & 0 \\
\hline$\Theta^{i}, \eta^{i}$ & 1 & $-\frac{1}{2}$ & 0 \\
\hline$p$ & -4 & 0 & 0 \\
\hline$\Theta_{p}, \eta_{p}$ & -4 & -1 & 0 \\
\hline$\beta_{a}$ & 1 & $-\frac{1}{2}$ & 1 \\
\hline$\wp$ & -4 & 0 & -1 \\
\hline
\end{tabular}

on the quartic K3, with the bundle $V$ defined by the sequence (2.10) with $m=4$ and $\left\{n_{a}\right\}=\{1,1,1,1\}$. There is a boundary superpotential coupling $\int d \theta \beta_{a} F^{a}(\phi) \wp$. The table at left displays the gauge charges, R-charge, and boundary global charges of all of the relevant fields. The bulk vector is neutral under all of these transformations.

Table 1: The charges of the relevant fields.

In the GLSM for this simple monad on K3, our supercharge takes the form

$$
\begin{aligned}
& Q^{\dagger}=Q_{\mathrm{bulk}}^{\dagger}+Q_{\partial}^{\dagger} \\
& =\int d x\left\{\nabla_{0} \phi_{i} \Theta^{i \dagger}+\nabla_{1} \phi_{i} \eta^{i \dagger}+2 p \partial_{i} G \eta^{i}+\nabla_{0} p \Theta_{p}^{\dagger}+\nabla_{1} p \eta_{p}^{\dagger}+2 G \eta_{p}\right. \\
& +\lambda_{+}\left(-\partial_{+} \sigma+\frac{1}{\sqrt{2}}\left(i D+\frac{v_{+-}}{\sqrt{2}}\right)\right)+\lambda_{-}\left(\partial_{-} \sigma^{\dagger}-\frac{1}{\sqrt{2}}\left(i D-\frac{v_{+-}}{\sqrt{2}}\right)\right) \\
& \left.\quad+\delta(\partial \Sigma)\left(\wp \beta_{a} F^{a}(\phi)+\eta_{p}^{\dagger} p\right)\right\} .
\end{aligned}
$$

- We will only be interested in operators which are invariant under the gauged special boundary symmetry (so that they map states in the charge-one sector back into the same sector). Furthermore, if we are not going to restrict ourselves to a phase of the theory where the bulk $U(1)$ is higgsed and integrate out the bulk gauge field, we must throw away operators which are not $U(1)$ invariant. We want to find the spectrum of massless spacetime fields. The fermionic parts of such multiplets arise from Ramond vertex operators whose internal parts have R-charge 0 or 1 . The former map via spectral flow to NS-sector operators of conformal weight 0 and hence lead to spacetime vectors. Ramond states of unit R-charge flow spectrally to NS operators of unit dimension which give the scalar components of spacetime chiral multiplets.

- The equation of motion of the boundary vector field $a_{0}$ is imposed as an operator equation of motion. So we impose the boundary Gauss' Law,

$$
: \beta_{a}^{\dagger} \beta_{a}:-: \wp^{\dagger} \wp:= \pm 1
$$


where the \pm depends on the orientation of the boundary component, on our operators.

- In order to be boundary-symmetry-invariant, an operator has to contain an even number of boundary fields. Operators without any boundary fields are just bulk operators restricted to the boundary and we ignore them. Included among these is the identity operator, which creates the center-of-mass vector. We claim that operators containing four or more boundary fields do not create any states beyond those made by operators bilinear in the fiber fields. We can see this by making the state-operator correspondence more explicit as follows.

The only subtlety involved is the vacuum degeneracy (present even in the NS sector) arising from the boundary fermion and boson zero modes. To resolve this, we choose a reference state,

$$
|0\rangle_{+} \otimes|0\rangle_{-}
$$

Here $|0\rangle_{ \pm}$are vacua of the two ends of the string satisfying

$$
0=\beta_{a}|0\rangle_{-}=\wp^{\dagger}|0\rangle_{-}=\beta_{a}^{\dagger}|0\rangle_{+}=\wp|0\rangle_{+}
$$

So we have arbitarily picked out a state which is not in the boundary Hilbert space of the ends of the string because it does not satisfy the boundary charge projection. The state corresponding to an operator is obtained by acting with that operator on this vacuum and projecting onto the subspace satisfying the charge projection. For example, an operator of the form $\beta_{a}^{\dagger} \beta_{b}$ makes the state with the - end of the string in sector $a$, and the + end of the string in sector $b$. Acting on this state, the only independent boundary operators invariant under the boundary symmetry are of the form

$$
\wp^{\dagger} \beta^{\dagger}, \wp^{\dagger} \wp, \beta^{\dagger} \beta, \wp \beta .
$$

Operators which are least quartic in the fiber fields are made by acting with one of these operators again. Using the charge projection (6.3) this will always give zero or a state created by a quadratic operator.

- It is convenient to divide up $Q^{\dagger}$ into

$$
Q^{\dagger}=Q_{0}^{\dagger}+Q_{1}^{\dagger}
$$

where $Q_{0}^{\dagger}$ includes only the parts depending on derivatives of bulk fields. By a zig-zag argument of the type appearing in [32] we can compute the cohomology of $Q^{\dagger}$ by 
computing the cohomology of $Q_{1}^{\dagger}$ in the cohomology of $Q_{0}^{\dagger}$. This tells us that we can leave out any non-holomorphic dependence on $\phi$ and we can leave out $\Theta$ 's and their daggers because any operator with $\Theta$ - or $\phi^{\dagger}$-dependence is a $Q^{\dagger}$ descendant.

- We discard the vector multiplet from our cohomology calculation. It is massive at large $|r|$, where its effect is to impose Gauss' law and its supersymmetric completions. Combined with the fact that the result of this calculation is independent of worldsheet FI terms (which are $Q^{\dagger}$ descendants), this means that we may safely neglect it. A more rigorous justification for this awaits future work.

- Operators containing the bulk field $p$, but not containing its partner $\eta_{p}^{\dagger}$ are in the image of the supercharge because of the term

$$
\delta(\partial \Sigma) \eta_{p}^{\dagger} p
$$

added to the supercharge to solve the Warner problem.

Schematically, the structure of the action of $Q^{\dagger}$ on the pertinent operators is:

$$
0 \rightarrow\left\{R_{(l)}^{a}(\phi) \beta_{a}^{\dagger} \wp^{\dagger}\right\} \rightarrow\left\{\begin{array}{l}
S_{(l+3)}(\phi) \wp^{\dagger} \wp \\
S_{(l+3)}^{a b}(\phi) \beta_{a}^{\dagger} \beta_{b}
\end{array}\right\} \rightarrow\left\{T_{(l+6)}^{a}(\phi) \beta_{a} \wp\right\} \rightarrow 0
$$

where the notation means that $R_{(l)}^{a}(\phi)$ is a homogeneous polynomial in $\phi$ of degree $l$ (and likewise for $S$ s and $T \mathrm{~s})$.

In order that these operators be gauge invariant we need $l=-3$. This means that the first node is trivial, and at the second node the polynomials are just constants. At the third node, the polynomials are cubic and these operators are exactly of the form of marginal deformations of the superpotential. Accordingly, they have R-charge 1. The image of $Q^{\dagger}$ from the R-charge zero operators at the previous node consists of operators of the form

$$
R_{a b} F^{a}(\phi) \beta_{b} \wp
$$

where $R_{a b}$ is a constant and $F^{a}$ is the section of $\bigoplus \mathcal{O}\left(n_{a}\right)$ appearing in the boundary superpotential. This tells us that in $Q^{\dagger}$ cohomology,

$$
T_{(3)}^{a}(\phi) \simeq T_{(3)}^{a}+R_{a b} f^{b}(\phi)
$$

Since there are twenty independent degree three monomials in four variables, we find $4(20-4)=64$ elements of cohomology at R-charge 1 from this part of the $Q^{\dagger}$ complex. 
At R-charge 0 , the only operator in $\operatorname{ker}\left(\mathrm{Q}^{\dagger}\right)$ is

$$
: \beta_{a}^{\dagger} \beta_{a}:-: \wp^{\dagger} \wp:=j_{s}
$$

which by the boundary Gauss' law (6.3) is the identity operator which creates (the fermion partner of) the center-of-mass vector field.

We note here that the brane worldvolume Higgs mechanism has a very natural implementation in this complex. Cohomology can appear at adjacent nodes which have R-charge 0 and 1 respectively, resulting in a new vector field and a new charged multiplet descending to zero mass.

Note that this framework could as easily have been applied directly to the largeradius phase of the theory. In that case, we would have simply set $p=0$ to its vacuum value and ignored its massive fluctuations. One finds the same operators representing the cohomology, and the calculation essentially reduces to the classical mathematics of deformation theory. It would be interesting to perform an analogous calculation directly in the Landau-Ginzburg effective field theory, after integrating out the $p$ field and gauge multiplet.

\section{Consequences of the index theorem}

For the Chern-classes of the $S U(3)$ bundle $V$ one finds $c_{1}(V)=0$ and $c_{2}(V)=24$. A theorem of Mukai [54] tells us that the dimension of the moduli space of such a bundle is related directly to the index of $Q^{\dagger}$, and in this example has (quaternionic) dimension 64. Therefore, one expects the brane spectrum to include 64 massless hypermultiplets, in agreement with our result.

The index of $Q^{\dagger}$ is not directly related to the dimension of the brane moduli space for branes on Calabi-Yau threefolds. In those examples, the index of $Q^{\dagger}$ is still invariant under smooth deformations of the closed string and open string moduli, but there are adjacent nodes in the complex representing scalar states which may pair up with each other while preserving the index of the $Q^{\dagger}$ complex. Note that the index of $Q^{\dagger}$ is invariant under addition of brane-antibrane pairs in the linear sigma model. 


\section{Future Directions}

Many interesting issues arise in the study of D-branes on Calabi-Yau spaces, and our formalism might be usefully extended to address a number of them. Here, we close by mentioning several subjects for future exploration:

- One expects that generic branes will cross lines of marginal stability in the $(r, \theta)$ plane. While a mechanism for implementing brane decays in the linear model was discussed in $\S 5.3$, it would be very interesting to derive the form of these loci of marginal stability directly in the linear model. This would presumably involve a direct calculation of the relevant spacetime central charge.

- The physics of D-branes at singular points in their moduli space (where even the boundary CFT becomes ill-defined) should be tractable in this approach. For closed strings, the new non-compact branches which arise in the linear model at singular points in moduli space were shown in e.g. [13] to allow one to reproduce detailed calculations about divergent terms in the spacetime effective action. A similar story may well arise here.

- A related question: At certain loci in moduli space, wrapped D-branes become massless spacetime states. This happens for instance at the mirror conifold point in the Kähler moduli space of the quintic, where the wrapped D6 brane becomes massless. What is the behavior of the worldvolume theory on the brane in such a limit? What happens as one makes extremal transitions to new branches of moduli space where such wrapped branes become fundamental string states?

- We have confined ourselves to discussing $B$-type branes in this paper; but similar methods could work for $A$-type branes as well (for earlier work in this direction see [14,15], for later work in this direction see [37]). As discussed in [18, 19, 55] there are expected to be intricate, disc-instanton generated superpotentials for $A$-type branes. It would be interesting to formulate a linear sigma model description of such branes in which the instanton sum was computable. In a somewhat analogous problem with more supersymmetry, Morrison and Plesser did succeed in reproducing closed string instanton effects directly in the linear sigma model [36]. For recent work in this direction, see [56].

- Finally, any microscopically consistent model with wrapped, space-filling D-branes will have to include orientifolds (or anti-branes) as well to cancel $R R$ tadpoles. It will be interesting to study new phenomena that arise in generalizing this kind of worldsheet description to models with orientifolds and/or antibranes. 


\section{Acknowledgements}

We are grateful to Jacques Distler, Kentaro Hori, Greg Moore, Michael Peskin, Joe Polchinski, Eva Silverstein, Lenny Susskind and Nick Warner for helpful discussions. Some discussions of boundary conditions were originally developed in unpublished work by Thiagarajan Jayaraman, A.L., Hirosi Ooguri and Tapobrata Sarkar. They have since appeared (with the absent authors' blessings) in [15]. A.L. would like to thank the other authors and Suresh Govindarajan for collaboration and discussions. Parts of this work were undertaken while S.K. and A.L. enjoyed the hospitality of the Aspen Center for Physics and the Institute for Theoretical Physics at Santa Barbara. This work was supported in part by the DOE under contract DE-AC03-76SF00515. The work of S.K. is also supported by a David and Lucile Packard Foundation Fellowship for Science and Engineering, an Alfred P. Sloan Foundation Fellowship, and National Science Foundation grant PHY00-97915. The work of J.M. was supported in part by the Department of Defense NDSEG Fellowship program.

\section{Appendix A. Transformation properties of bulk supermultiplets}

Throughout this paper we use $Q=\frac{Q_{+}-Q_{-}}{\sqrt{2}}$ and its conjugate as the generators of the unbroken B-type supersymmetry. $S=\frac{Q_{+}+Q_{-}}{\sqrt{2}}$ and its conjugate generate the supersymmetry transformations broken by the boundary theory.

The $(2,2)$ algebra is

$$
\begin{gathered}
\left\{Q_{ \pm}, Q_{ \pm}\right\}=0 \quad\left\{Q_{ \pm}, Q_{\mp}\right\}=0 \\
\left\{Q_{ \pm}, Q_{ \pm}^{\dagger}\right\}=2 P_{ \pm} \\
\left\{Q_{+}, Q_{-}^{\dagger}\right\}=2 Z \quad\left\{Q_{-}, Q_{+}^{\dagger}\right\}=2 Z^{\dagger} \\
{\left[Q_{ \pm}, P_{\mp}\right]=-i \sqrt{2} q \lambda_{\mp}^{\dagger} \quad\left[Q_{ \pm}^{\dagger}, P_{\mp}\right]=-i \sqrt{2} q \lambda_{\mp}}
\end{gathered}
$$

where $Z$ acts by multiplication by $q \sigma^{\dagger}$ on a field of charge $q$ and $P_{ \pm}=-i D_{ \pm}$is the gauge covariant momentum (but does not contain $\sigma \mathrm{s}$ ). Note that $\left[\nabla_{ \pm}, Q\right]=\left[\nabla_{ \pm}, Q^{\dagger}\right]=0$.

In the following, our supercharges act by graded commutation. The $(2,2)$ supersymmetry transformations of the fields in a chiral multiplet, $\phi$, of $U(1)$ gauge charge $a$ in Wess-Zumino gauge are 


$$
\begin{aligned}
& Q \phi=-i \Theta \quad Q^{\dagger} \phi=0 \quad Q \phi^{\dagger}=0 \quad Q^{\dagger} \phi^{\dagger}=-i \Theta^{\dagger} \\
& S \phi=-i \eta \quad S^{\dagger} \phi=0 \quad S \phi^{\dagger}=0 \quad S^{\dagger} \phi^{\dagger}=-i \eta^{\dagger} \\
& Q \Theta=0 \quad Q^{\dagger} \Theta=2 \nabla_{0} \phi \quad Q \Theta^{\dagger}=2 \nabla_{0} \phi^{\dagger} \quad Q^{\dagger} \Theta^{\dagger}=0 \\
& S \Theta=-F \quad S^{\dagger} \Theta=2 \nabla_{1} \phi \quad S \Theta^{\dagger}=2\left(\nabla_{1} \phi\right)^{\dagger} \quad S^{\dagger} \Theta^{\dagger}=-F^{\dagger} \\
& Q \eta=F \quad Q^{\dagger} \eta=2 \tilde{\nabla}_{1} \phi \quad Q \eta^{\dagger}=2\left(\tilde{\nabla}_{1} \phi\right)^{\dagger} \quad Q^{\dagger} \eta^{\dagger}=F^{\dagger} \\
& S \eta=0 \quad S^{\dagger} \eta=2 \tilde{\nabla}_{0} \phi \quad S \eta^{\dagger}=2 \tilde{\nabla}_{0} \phi^{\dagger} \quad S^{\dagger} \eta^{\dagger}=0 \\
& Q F=0 \quad Q F^{\dagger}=2 i\left(\left(\tilde{\nabla}_{1} \Theta\right)^{\dagger}-\nabla_{0} \eta^{\dagger}\right)+2 q \phi^{\dagger}\left(\lambda_{+}+\lambda_{-}\right) \\
& Q^{\dagger} F=2 i\left(\tilde{\nabla}_{1} \Theta-\nabla_{0} \eta\right)+2 q \phi\left(\lambda_{+}^{\dagger}+\lambda_{-}^{\dagger}\right) \quad Q^{\dagger} F^{\dagger}=0 \\
& S F=0 \quad S F^{\dagger}=2 i\left(\tilde{\nabla}_{0} \Theta^{\dagger}-\left(\nabla_{1} \eta\right)^{\dagger}\right)+2 q \phi^{\dagger}\left(\lambda_{+}-\lambda_{-}\right) \\
& S^{\dagger} F=2 i\left(\tilde{\nabla}_{0} \Theta-\nabla_{1} \eta\right)+2 q \phi\left(\lambda_{+}^{\dagger}-\lambda_{-}^{\dagger}\right) \quad S^{\dagger} \Theta^{\dagger}=-F^{\dagger}
\end{aligned}
$$

The vector multiplet fields transform as:

$$
\begin{aligned}
& Q \sigma=i \lambda_{+}^{\dagger} \quad Q^{\dagger} \sigma=-i \lambda_{-} \quad Q \sigma^{\dagger}=-i \lambda_{-}^{\dagger} \quad Q^{\dagger} \sigma^{\dagger}=i \lambda_{+} \\
& S \sigma=i \lambda_{+}^{\dagger} \quad S^{\dagger} \sigma=i \lambda_{-} \quad S \sigma^{\dagger}=i \lambda_{-}^{\dagger} \quad S^{\dagger} \sigma^{\dagger}=i \lambda_{+} \\
& Q \lambda_{+}=-\partial_{+} \sigma^{\dagger}-\frac{1}{\sqrt{2}}\left(i D-\frac{v_{+-}}{\sqrt{2}}\right) \quad Q^{\dagger} \lambda_{+}^{\dagger}=-\partial_{+} \sigma+\frac{1}{\sqrt{2}}\left(i D+\frac{v_{+-}}{\sqrt{2}}\right) \\
& Q^{\dagger} \lambda_{+}=0 \quad S^{\dagger} \lambda_{+}=0 \quad Q \lambda_{+}^{\dagger}=0 \quad S \lambda_{+}^{\dagger}=0 \\
& S \lambda_{+}=-\partial_{+} \sigma^{\dagger}+\frac{1}{\sqrt{2}}\left(i D-\frac{v_{+-}}{\sqrt{2}}\right) \quad S^{\dagger} \lambda_{+}^{\dagger}=-\partial_{+} \sigma-\frac{1}{\sqrt{2}}\left(i D+\frac{v_{+-}}{\sqrt{2}}\right) \\
& Q \lambda_{-}=\partial_{-} \sigma+\frac{1}{\sqrt{2}}\left(i D+\frac{v_{+-}}{\sqrt{2}}\right) \quad Q^{\dagger} \lambda_{-}^{\dagger}=\partial_{-} \sigma^{\dagger}-\frac{1}{\sqrt{2}}\left(i D-\frac{v_{+-}}{\sqrt{2}}\right) \\
& Q^{\dagger} \lambda_{-}=0 \quad S^{\dagger} \lambda_{-}=0 \quad Q \lambda_{-}^{\dagger}=0 \quad S \lambda_{-}^{\dagger}=0 \\
& S \lambda_{-}=-\partial_{-} \sigma+\frac{1}{\sqrt{2}}\left(i D+\frac{v_{+-}}{\sqrt{2}}\right) \quad S^{\dagger} \lambda_{-}^{\dagger}=-\partial_{-} \sigma^{\dagger}-\frac{1}{\sqrt{2}}\left(i D-\frac{v_{+-}}{\sqrt{2}}\right) \\
& Q v_{+-}=-i\left(\partial_{+} \lambda_{-}^{\dagger}+\partial_{-} \lambda_{+}^{\dagger}\right) \quad Q^{\dagger} v_{+-}=-i\left(\partial_{+} \lambda_{-}+\partial_{-} \lambda_{+}\right) \\
& S v_{+-}=-i\left(\partial_{+} \lambda_{-}^{\dagger}-\partial_{-} \lambda_{+}^{\dagger}\right) \quad S^{\dagger} v_{+-}=-i\left(\partial_{+} \lambda_{-}-\partial_{-} \lambda_{+}\right) \\
& Q i \sqrt{2} D=i\left(\partial_{+} \lambda_{-}^{\dagger}-\partial_{-} \lambda_{+}^{\dagger}\right) \quad Q^{\dagger} i \sqrt{2} D=-i\left(\partial_{+} \lambda_{-}-\partial_{-} \lambda_{+}\right) \\
& S i \sqrt{2} D=i\left(\partial_{+} \lambda_{-}^{\dagger}+\partial_{-} \lambda_{+}^{\dagger}\right) \quad S^{\dagger} i \sqrt{2} D=-i\left(-\partial_{+} \lambda_{-}+\partial_{-} \lambda_{+}\right)
\end{aligned}
$$




\section{Appendix B. Boundary superspace}

We can exponentiate the supersymmetry transformations to define a superspace formalism. We use the same bulk superspace coordinates as in [9]. In this language the action of supersymmetry on superfields is generated by:

$$
\begin{aligned}
\delta \Phi & =\left(\epsilon^{\alpha} Q_{\alpha}-\bar{\epsilon}^{\alpha} Q_{\alpha}^{\dagger}\right) \Phi \\
& =\left(-\epsilon_{-} Q_{+}+\epsilon_{+} Q_{-}+\bar{\epsilon}_{-} Q_{+}^{\dagger}-\bar{\epsilon}_{+} Q_{-}^{\dagger}\right) \Phi
\end{aligned}
$$

where $\epsilon^{\alpha}$ is the Grassman parameter; the spinor indices are raised and lowered by the antisymmetric tensor $\epsilon_{\alpha \beta}$ as in [9]. Recall that

$$
\begin{aligned}
& Q_{+}=\frac{\partial}{\partial \theta^{+}}+i \bar{\theta}^{+} \partial_{+} \\
& Q_{-}=\frac{\partial}{\partial \theta^{-}}+i \bar{\theta}^{-} \partial_{-} \\
& Q_{+}^{\dagger}=-\frac{\partial}{\partial \bar{\theta}^{+}}-i \theta^{+} \partial_{+} \\
& Q_{-}^{\dagger}=-\frac{\partial}{\partial \bar{\theta}^{-}}-i \theta^{-} \partial_{-}
\end{aligned}
$$

where

$$
\partial_{ \pm}=\partial_{0} \pm \partial_{1}
$$

We can similarly define superspace derivatives:

$$
\begin{aligned}
& D_{ \pm}=\frac{\partial}{\partial \theta^{ \pm}}-i \bar{\theta}^{ \pm} \partial_{ \pm} \\
& \bar{D}_{ \pm}=-\frac{\partial}{\partial \bar{\theta}^{ \pm}}+i \theta^{ \pm} \partial_{ \pm}
\end{aligned}
$$

We wish to preserve as symmetries those transformations for which $\epsilon_{+}=\epsilon_{-}$. Define:

$$
\begin{aligned}
& \epsilon=\frac{1}{\sqrt{2}}\left(\epsilon_{+}+\epsilon_{-}\right) \\
& \tilde{\epsilon}=\frac{1}{\sqrt{2}}\left(\epsilon_{+}-\epsilon_{-}\right)
\end{aligned}
$$

and set $\tilde{\epsilon}=0$. Then

$$
\delta \Phi=\left(\epsilon \frac{Q_{-}-Q_{+}}{\sqrt{2}}+\bar{\epsilon} \frac{Q_{+}^{\dagger}-Q_{-}^{\dagger}}{\sqrt{2}}\right) \Phi
$$

We can define

$$
\theta=\frac{\theta^{+}-\theta^{-}}{\sqrt{2}}
$$


and

$$
Q=\frac{Q_{+}-Q_{-}}{\sqrt{2}}=\frac{\partial}{\partial \theta}+i \bar{\theta} \partial_{0} \quad Q^{\dagger}=\frac{Q_{+}^{\dagger}-Q_{-}^{\dagger}}{\sqrt{2}}=-\frac{\partial}{\partial \bar{\theta}}-i \theta \partial_{0}^{\dagger}
$$

so that

$$
\delta \Phi=\left(\epsilon Q-\bar{\epsilon} Q^{\dagger}\right) \Phi
$$

Similarly, we can define superspace derivatives:

$$
\begin{aligned}
& D=\frac{D_{+}-D_{-}}{\sqrt{2}}=\frac{\partial}{\partial \theta}-i \bar{\theta} \partial_{0} \\
& \bar{D}=\frac{\bar{D}_{+}-\bar{D}_{-}}{\sqrt{2}}=-\frac{\partial}{\partial \bar{\theta}}+i \theta \partial_{0} .
\end{aligned}
$$

\section{Appendix C. The tensor formula}

Here we state a formula for the tensor product of two sheaves. The support of this product sheaf is the intersection of the supports of the original sheaves.

Take a complex

$$
0 \rightarrow V_{0} \stackrel{d_{1}}{\longrightarrow} V_{1} \stackrel{d_{2}}{\longrightarrow} \cdots \stackrel{d_{m}}{\longrightarrow} V_{m} \rightarrow 0
$$

whose cohomology is a sheaf $E$, and a complex

$$
0 \rightarrow W_{0} \stackrel{\tilde{d}_{1}}{\longrightarrow} W_{1} \stackrel{\tilde{d}_{2}}{\longrightarrow} \cdots \stackrel{\tilde{d}_{n}}{\longrightarrow} W_{n} \rightarrow 0
$$

whose cohomology is a sheaf $F$. Then a complex whose cohomology is $E \otimes F$ is:

$$
0 \rightarrow V_{0} \otimes W_{0} \stackrel{D_{1}}{\longrightarrow} V_{0} \otimes W_{1} \oplus V_{1} \otimes W_{0} \stackrel{D_{2}}{\longrightarrow} V_{0} \otimes W_{2} \oplus V_{1} \otimes W_{1} \oplus V_{2} \otimes W_{0} \stackrel{d_{3}}{\longrightarrow} \cdots \rightarrow 0
$$

where

$$
D_{r+s+1}(a \otimes b)=d_{r+1}(a) \otimes b+(-1)^{r} a \otimes \tilde{d}_{s+1}(b)
$$

for $a \in V_{r}$ and $b \in W_{s}$.

This formula is well known to mathematicians, to whom, however, the minus signs in (C.4) seem mysterious (c.f. p. 431 of [57]). Rather than proving this directly as a mathematical statement, we can give a physical interpretation. Consider two sets of fiber

fields $\beta, \wp$ and $\tilde{\beta}, \tilde{\wp}$ which transform under two independent boundary symmetries $U(1)_{1,2}$. The $\beta, \wp$ fields are linear model fields satisfying the correct deformed chiral constraints to have cohomology equal to $E$. That is

$$
\begin{aligned}
\left\{Q^{\dagger}, \beta_{2 n}\right\} & =d_{2 n-1} \wp_{2 n-1}^{\dagger} \\
{\left[Q^{\dagger}, \wp_{2 n+1}^{\dagger}\right] } & =d_{2 n} \beta_{2 n} .
\end{aligned}
$$


Similarly, $\tilde{\beta}, \tilde{\wp}$ satisfy the contraints:

$$
\begin{aligned}
\left\{Q^{\dagger}, \tilde{\beta}_{2 n}\right\} & =\tilde{d}_{2 n-1} \tilde{\wp}_{2 n-1}^{\dagger} \\
{\left[Q^{\dagger}, \tilde{\wp}_{2 n+1}^{\dagger}\right] } & =\tilde{d}_{2 n} \tilde{\beta}_{2 n}
\end{aligned}
$$

in order to describe $F$. We can combine $\beta_{k}, \wp_{k}^{\dagger}$ into an object $\gamma_{m}$ which is even(odd) when $m$ is odd(even). If the left endpoint lives in $E \otimes F$, the fields for both $E$ and $F$ should be excited. Thus we perform two boundary charge projections, each onto charge sector +1 .

To see that this is equivalent to the complex (C.3) with exterior derivative $D$, examine variables invariant under the combination $U(1)_{1}-U(1)_{2}$. The gauge invariant combinations which satisfy deformed chiral constraints off shell are $\beta \tilde{\beta}, \beta \tilde{\wp}^{\dagger}, \wp^{\dagger} \tilde{\beta}, \wp^{\dagger} \tilde{\wp}^{\dagger}$. These combinations all have charge +1 under the remaining symmetry $\frac{1}{2}\left(U(1)_{1}+U(1)_{2}\right)$, and satisfy:

$$
\left[Q^{\dagger}, \gamma_{m} \tilde{\gamma}_{n}\right]=d_{m-1}\left(\gamma_{m-1}\right) \tilde{\gamma}_{n}+(-1)^{m-1} \gamma_{m} \tilde{d}_{n-1}\left(\tilde{\gamma}_{n-1}\right) .
$$

Thus, the singlets of the confined gauge group $U(1)_{1}-U(1)_{2}$ transform under supersymmetry as fibers in the complex defining $E \otimes F$. 


\section{References}

[1] B.R. Greene, "D-brane topology changing transitions," Nucl. Phys. B525 (1998) 284, hep-th/9711124.

[2] T. Muto, "D-branes at orbifolds and topology change," Nucl. Phys. 521 (1998) 183, hep-th/9711090.

[3] S. Kachru, A. Lawrence and E. Silverstein, "On the matrix description of Calabi-Yau compactifications," Phys. Rev. Lett. 80 (1998) 2996, hep-th/9712223.

[4] S. Kachru and J. McGreevy, "Supersymmetric three-cycles and supersymmetry breaking," Phys. Rev. D61 (2000) 026001, hep-th/9908135.

[5] I. Brunner, M.R. Douglas, A. Lawrence and C. Römelsberger, "D-branes on the Quintic," JHEP 0008 (2000) 015, hep-th/9906200.

[6] M.R. Douglas, B. Fiol and C. Römelsberger, "Stability and BPS branes," hepth/0002037.

[7] F. Denef, "Supergravity Flows and D-brane Stability," JHEP 0008 (2000) 050, hepth/0005049;

F. Denef, B. Greene and M. Raugas, "Split Attractor Flows and the Spectrum of BPS

D-branes on the Quintic," JHEP 0105 (2001) 012, hep-th/0101135;

F. Denef, “(Dis)assembling Special Lagrangians," hep-th/0107152.

[8] M.R. Douglas, B. Fiol and C. Römelsberger, "The spectrum of BPS branes on a noncompact Calabi-Yau," hep-th/0003263.

[9] E. Witten, "Phases of $\mathrm{N}=2$ theories in two dimensions," Nucl. Phys. B403 (1993) 159, hep-th/9301042.

[10] P. Aspinwall, B. Greene and D. Morrison, "Calabi-Yau moduli space, mirror manifolds and space-time topology change in string theory," Nucl. Phys. B416 (1994) 414, hepth/9309097.

[11] T.-M. Chiang and B.R. Greene, "Phases of mirror symmetry," Strings '95 proceedings Future Perspectives in String Theory (World Scientific, New Jersey) 1996; hepth/9509049.

[12] B. Greene, C. Vafa and N. Warner, "Calabi-Yau manifolds and renormalization group flows," Nucl. Phys. B324 (1989) 371;

E. Martinec, "Criticality, Catastrophes and Compactifications," in Brink, L. (ed.) et al., Physics and Mathematics of Strings 383.

[13] E. Silverstein and E. Witten, "Criteria for conformal invariance of $(0,2)$ models," Nucl. Phys. 444 (1995) 161, hep-th/9503212.

[14] K. Hori, A. Iqbal and C. Vafa, "D-Branes and mirror symmetry," hep-th/0005247.

[15] S. Govindarajan, T. Jayaraman, and T. Sarkar, "On D-branes from gauged linear sigma models," hep-th/0007075. 
[16] K. Becker, M. Becker and A. Strominger, "Five-branes, membranes and nonperturbative string theory," Nucl. Phys. B456 (1995) 130, hep-th/9507158.

[17] H. Ooguri, Y. Oz and Z. Yin, "D-branes on Calabi-Yau spaces and their mirrors," Nucl. Phys. B477 (1996) 407, hep-th/9606112.

[18] S. Kachru, S. Katz, A. Lawrence and J. McGreevy, "Open String Instantons and Superpotentials," Phys. Rev. D62 (2000) 026001, hep-th/9912151.

[19] S. Kachru, S. Katz, A. Lawrence and J. McGreevy, "Mirror symmetry for open strings," hep-th/0006047.

[20] D.-E. Diaconescu and M.R. Douglas, "D-branes on stringy Calabi-Yau manifolds," hep-th/0006224.

[21] J. Distler and S. Kachru, “(0,2) Landau-Ginzburg Theory,” Nucl. Phys. B413 (1993) 213, hep-th/9309110.

[22] Talk given by S. Kachru at Strings 2000, http://feynman.physics.1sa.umich.edu/cgibin/s2ktalk.cgi?kachru

[23] P. Mayr, "Phases of supersymmetric D-branes on Kaehler manifolds and the McKay correspondence," hep-th/0010223.

[24] K. Hori, "Linear models of supersymmetric D-branes," hep-th/0012179.

[25] S. Govindarajan, T. Jayaraman, "D-branes, exceptional sheaves and quivers on CalabiYau manifolds," Nucl. Phys. B600 (2001) 457, hep-th/0010196; "Boundary fermions, coherent sheaves and D-branes on Calabi-Yau manifolds," hep-th/0104126.

[26] S. Hellerman and J. McGreevy, "Linear sigma model toolshed for D-brane physics," hep-th/0104100.

[27] J. Distler, "Notes on $(0,2)$ superconformal field theories," Trieste HEP Cosmology 1994:0322-351, hep-th/9502012.

[28] P. Fendley, H. Saleur and N.P. Warner, "Exact solution of a massless scalar field with a relevant boundary interaction," Nucl. Phys. B430 (1994) 577, hep-th/9406125;

J.A. Harvey, D. Kutasov and E.J. Martinec, "On the relevance of tachyons," hepth/0003101.

[29] D. Gepner, "Exactly Solvable String Compactifications on Manifolds of $S U(N)$ Holonomy," Phys. Lett. B199 (1987) 380.

[30] N. Seiberg, Phys. Lett. B318, 469 (1993), hep-ph/9309335.

[31] E. Witten, "On the Landau-Ginzburg description of $\mathrm{N}=2$ minimal models," Int. J. Mod. Phys. A9 (1994) 4783, hep-th/9304026.

[32] S. Kachru and E. Witten, "Computing the Complete Massless Spectrum of a LandauGinzburg Orbifold," hep-th/9307038.

[33] E. Witten, "Mirror manifolds and topological field theory," in Mirror Symmetry I, S.-T. Yau (ed.), American Mathematical Society (1998), hep-th/9112056.

[34] W. Lerche, C. Vafa and N.P. Warner, "Chiral rings in $\mathcal{N}=2$ superconformal theories," Nucl. Phys. B324 (1989) 427. 
[35] C. Lazariou, "String field theory and brane superpotentials," hep-th/0107162;

A. Tomasiello, "A-infinity structure and superpotentials," hep-th/0107195.

[36] D. Morrison and M.R. Plesser, "Summing the Instantons: Quantum Cohomology and Mirror Symmetry in Toric Varieties," Nucl. Phys. B440 (1995) 279, hep-th/9412236.

[37] S. Hellerman and J. McGreevy, work in progress.

[38] N. Warner, "Supersymmetry in boundary integrable models," Nucl. Phys. B450 (1995) 663, hep-th/9506064

[39] R. I. Nepomechie, "The boundary N = 2 supersymmetric sine-Gordon model," Phys. Lett. B 516, 376 (2001); hep-th/0106207, "Boundary S matrices with $\mathrm{N}=2$ supersymmetry," Phys. Lett. B 516, 161 (2001), hep-th/0106223.

[40] J. Polchinski, String Theory, two vols., Cambridge (1999).

[41] M. Douglas, "D-branes, Categories and $\mathcal{N}=1$ Supersymmetry," hep-th/0011017.

[42] P. Aspinwall and A. Lawrence, "Derived Categories and Zero-Brane Stability," hepth/0104147.

[43] C. I. Lazaroiu, JHEP 0106, 052 (2001), hep-th/0102122; "Unitarity, D-brane dynamics and D-brane categories," hep-th/0102183; D.-E. Diaconescu, "Enhanced D-brane Categories from String Field Theory," hep-th/0104200; C. I. Lazaroiu, JHEP 0106, 064 (2001), hep-th/0105063; C. I. Lazaroiu, R. Roiban and D. Vaman, hep-th/0107063.

[44] P. Griffiths and J. Harris, Principles of Algebraic Geometry, John Wiley and Sons (1978) NY.

[45] D. Kutasov, G. Moore and M. Mariño, "Some exact results on tachyon condensation in string field theory," JHEP 0010 (2000) 045, hep-th/0009148.

[46] T. Gomez and E. Sharpe, "D-branes and Scheme Theory," hep-th/0008150.

[47] P. Seidel and R.P. Thomas, "Braid group actions on derived categories of coherent sheaves," math.AG/0001043; P.S. Aspinwall, "Some navigation rules for D-brane monodromy," hep-th/0102198; P. Horja, "Derived category automorphisms from mirror symmetry," math.AG/0103231.

[48] P. Candelas, X.C. de la Ossa, P.S. Green and L. Parkes, "A pair of Calabi-Yau manifolds as an exactly solvable superconformal field theory," Nucl. Phys. 359 (1991) 21.

[49] E. Witten and J. Bagger, "Quantization of Newton's Constant in Certain Supergravity Theories," Phys. Lett. B115 (1982) 202.

[50] E. Witten, "Small Instantons in String Theory," Nucl. Phys. B460 (1996) 541, hepth/9511030.

[51] P. Fayet, "Higgs Model and Supersymmetry," Nuovo Cim. A31 (1976) 626.

[52] D. Joyce, "On counting special lagrangian homology three spheres," hep-th/9907013.

[53] E. Sharpe, "Kähler cone substructure," Adv.Theor.Math.Phys. 2 (1999) 1441, hepth/9810064. 
[54] S. Mukai, "Symplectic structure of the moduli space of sheaves on an abelian or K3 surface," Invent. Math. 77 (1984) 101.

[55] M. Aganagic and C. Vafa, "Mirror Symmetry, D-branes and Counting Holomorphic Discs," hep-th/0012041;

M. Aganagic, A. Klemm and C. Vafa, "Disk Instantons, Mirror Symmetry and the Duality Web," hep-th/0105045.

[56] S. Govindarajan, T. Jayaraman and T. Sarkar, "Disc Instantons in Linear Sigma Models," hep-th/0108234; P. Mayr, "N = 1 mirror symmetry and open/closed string duality," hep-th/0108229.

[57] D. Eisenbud, Commutative Algebra with a View Toward Algebraic Geometry, Springer (1996). 